\title{
Strategies for molecular imaging dementia and neurodegenerative diseases
}

\author{
Bernhard J Schaller \\ Department of Neurosurgery, \\ University of Paris, Paris, France
}

Correspondence: Bernhard J Schaller Department of Neurosurgery, University of Paris, Paris, France Email skull_base_surgery@yahoo.de

\begin{abstract}
Dementia represents a heterogeneous term that has evolved to describe the behavioral syndromes associated with a variety of clinical and neuropathological changes during continuing degenerative disease of the brain. As such, there lacks a clear consensus regarding the neuropsychological and other constituent characteristics associated with various cerebrovascular changes in this disease process. But increasing this knowledge has given more insights into memory deterioration in patients suffering from Alzheimer's disease and other subtypes of dementia. The author reviews current knowledge of the physiological coupling between cerebral blood flow and metabolism in the light of state-of-the-art-imaging methods and its changes in dementia with special reference to Alzheimer's disease. Different imaging techniques are discussed with respect to their visualizing effect of biochemical, cellular, and/or structural changes in dementia. The pathophysiology of dementia in advanced age is becoming increasingly understood by revealing the underlying basis of neuropsychological changes with current imaging techniques, genetic and pathological features, which suggests that alterations of (neuro)vascular regulatory mechanisms may lead to brain dysfunction and disease. The current view is that cerebrovascular deregulation is seen as a contributor to cerebrovascular pathologies, such as stroke, but also to neurodegenerative conditions, such as Alzheimer's disease. The better understanding of these (patho)physiological mechanisms may open an approach to new interventional strategies in dementia to enhance neurovascular repair and to protect neurovascular coupling.
\end{abstract}

Keywords: imaging, cognitive impairment, Alzheimer's disease, cerebrovascular disease, neurovascular coupling

\section{Introduction}

Dementia represents a general term used to describe a chronic and substantial decline in two or more areas of cognitive function related to cellular and subcellular changes in the brain (Erkinjuntti et al 1997) underlining the multifactor origin of dementia syndrome (Pohjasvaara et al 2000). Imaging techniques (IT) supporting the diagnosis of different dementia subgroups of are moving from a negative, exclusionary role (American Academy of Neurology 1994) to one that reveals positive diagnostic, therapeutic, and/or prognostic value. FIT can offer an important impact in the treatment of dementia (Schaller et al 2003).

Cerebrovascular changes (CVC) represent a common symptom of the physiological aging process (Schaller 2004a, 2004b, 2005; Schaller et al 2004a, 2004b), and cerebrovascular risk factors are strongly associated with dementia including Alzheimer's disease (AD) (Cummings 1994; Desmond 1996; Skoog 1998). About $40 \%$ of the population who met the diagnostic criteria for probable AD also show markers for cerebrovascular disease (CVD) (Desmond 1996; Iadecola 2003; Jellinger et al 2003; Kleindienst et al 2004a, 2004b). In addition, different well recognized risk factors for CVD are strongly associated with $\mathrm{AD}$ and have elucidated the 
impact of cerebrovascular lesions on the development and progression of dementia in AD (Jellinger et al 2002, 2003) suggesting that there exists a relationship between elevated midlife blood pressure and late-life AD (Forette et al 1998). With the recent availability of several effective pharmaceutical agents for treatment of dementia undergoing clinical trials (Tang et al 2006), a new area will be entered emphasizing the increasing need to diagnose dementia in its more earlier stages (Zanetti 1998), so that new IT gain increased importance in last years (Hillman et al 1997; Paris 1997). FIT represent the potential to enable identification of more subtle pathological (sub)cellular changes earlier during the disease course (Buckner et al 2000; Bondi et al 2005). To better use these new diagnostic modalities, a thorough understanding of the physiological changes in the aging process and of the pathophysiological steps in dementia is needed.

\section{Physiological background as related to dementia \\ The coupling between neuronal activity and cerebral metabolism}

Current physiological-based hypotheses regarding the activity-/metabolism-coupling in different activated states of the brain (Figure 1), whether activated neurons in the brain significantly increase their consumption of oxygen (cerebral metabolic rate, $\mathrm{CMR}_{\mathrm{O} 2}$ ) or whether they exploit anaerobic metabolism for energy production, have been disputed (Schaller et al 2003). In particular, glutamate, released by activated neurons, would be the pivotal element coupling glucose consumption $\left(\mathrm{CMR}_{\mathrm{glc}}\right)$ to excitatory neuronal activity (NA) (Pellerin et al 1994) involving glutamate uptake into astrocytes, resulting in an increased intracellular $\mathrm{Na}^{+}$ concentration $\left(\left[\mathrm{Na}_{i}^{+}\right]\right)$and activation of the $\mathrm{Na}^{+} / \mathrm{K}^{+}$-ATPase (Chatton et al 2003; Bernardinelli 2004). Such increased metabolic demand linked to disruption of $\mathrm{Na}^{+}$homeostasis may activate glucose uptake and glycolysis in astrocytes

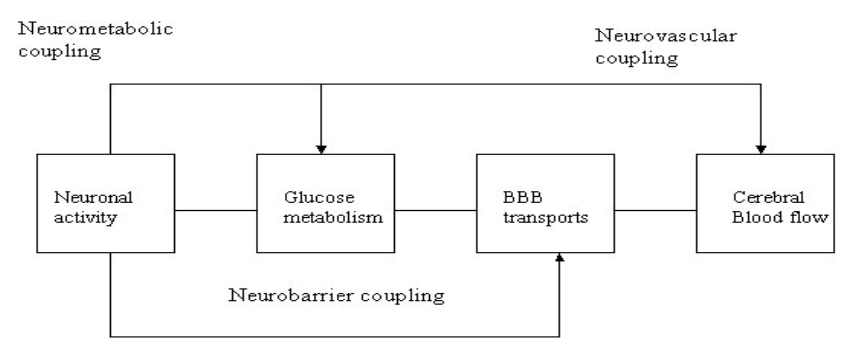

Figure I Neurobarrier coupling in the brain: The potential neurovascular and neurometabolic coupling (Leybaert 2005).

Abbreviation: BBB, blood-brain barrier.
(Chatton et al 2003). Therefore, astrocytes appear to function as a network for concentrated neurometabolic coupling through $\mathrm{Na}^{+}$gerneration and increased metabolic turnover (Bernardinelli et al 2004). Indeed, a stoichiometric relation has been reported between glutamate- and glucose-uptake by cultured astrocytes and likewise between glutamate cycling and $\mathrm{CMR}_{\mathrm{glc}}$ in mice cortex (Pellerin et al 1994). This proposed model is consistent with the view that during NA, glutamate uptake into astrocytes leads to increased $\mathrm{CMR}_{\mathrm{glc}}$ and lactate production, which can be subsequently used by neurons to meet their energy needs (Magistrettti 2000).

NA applies to a spectrum of energy requiring processes including action potential propagation, neurotransmitter release and up-take, vesicular recycling, and maintenance of membrane potential, providing a conceptual framework for the interpretation of new experimental data on the molecular aspects of cerebral energy metabolism (Tsacopoulos et al 1996). All these processes are involved in short-term neuronal information encoding, but the relative distribution of energy among them and how the different subclasses of neurons in a specific cortical region contribute to the overall energy consumption process remains an open question. At present, FIT identifies the location of changes in NA, but provides still no insight into the total NA involved in a process (Magistretti 2000; Magistretti et al 1999). In addition, the stoichiometric relationship between glutamate cycling and oxidative glucose metabolism argues for a quantitative coupling between NA and cerebral metabolism and would be in favor of metabolism as an index of NA (Magistretti et al 1999).

$\gamma$-Aminobutric acid (GABA) is the major inhibitory neurotransmitter in the central nervous system (CNS). GABA does not couple inhibitory NA with $\mathrm{CMR}_{\mathrm{glc}}$, as does glutamate for excitatory neurotransmission, and suggest that GABA-mediated synaptic transmission does not contribute directly to signals in IT based on deoxyglucose (Chatton 2003). In addition, anatomical and physiological arguments have been put forward to support the physiological concept that inhibition might involve lower metabolic demands (Magistretti 2000; Chatton 2003). Based on their reduced number, strategic position, and increased efficiency, inhibition provided by synapses from GABAergic interneurons in the cortex might simply involve less energy expenditure upon activation than the excitatory drive (Koistinaho 2003). Thus glutamate, by activating GABA neurons, could lead to an overall inhibitory NA in a specific cortical area; yet glutamate release and uptake by astrocytes would stimulate glycolysis in astrocytes and constitute a sufficient signal 
coding for increased activity of GABAergic neurons as well (Chatton et al 2003). It would therefore appear that the most relevant, and possibly sufficient imaging signal, linking NA (both excitatory and inhibitory) with metabolism is glutamate, which would mediate the appropriate metabolic coupling both for excitatory and inhibitory neurons (Magistretti 2000).

\section{The neurovascular coupling}

Experimental studies provide evidence for a cascade of physiological events for NVC whereby glutamate, leaking from active synapses, activates glutamate receptors on astrocyte membranes, leading to an elevation of intracellular $\mathrm{Ca}^{2+}$ $\left[\mathrm{Ca}_{\mathrm{i}}^{2+}\right]$, which in turn leads to the biochemical production and release of eicosanioids, which represent potent vasodilators (Bonvento et al 2002). Interruption of any stage of this cascade reduces the cerebral blood flow (CBF) response severely, without affecting the neural response (Magistretti 2000; Bonvento et al 2002).

Advances in this research field have demonstrated that release of the major excitatory neurotransmitter glutamate initiates physiologically diverse signaling processes between neurons and astrocytes, and that this signaling could be crucial for the occurrence of molecular imaging signals, although the neurovascular importance of these mechanisms is not clear (Bonvento et al 2002). Re-uptake of glutamate from the synaptic cleft into astrocytes is mediated by the specific highaffinity glial transporters GLAST (glial glutamate aspartate transporter) and GLT-1 (glutamate transporter 1), which are particularly enriched in brain areas with high glutamatergic activity and are essential to maintain glutamate levels below neurotoxicity limits (Schmidt et al 2004).

A positive linear correlation has been reported between the integrated electrophysiological activities, ie, summed evoked potentials (EP), at different frequencies of NA stimulation and CBF response (Ances et al 2000b), demonstrating that $\mathrm{CBF}$ response did not depend on NA intensity when duration was held constant (time-intensity separability) (Ances et al 2000b). However, mathematical modeling of the relationship between $\mathrm{CBF}$ and electrophysiological response to somatosensory stimulation revealed not only a linear but also a nonlinear component in this NVC (Ances et al 2000b). Indeed, changing the stimulus duration, but not the intensity, results in a different pattern of responses for $\mathrm{CBF}$ and somatosensory EP, each one exhibiting maximal amplitude for different stimulus durations (Ances et al 2000b). Moreover, activation-flow coupling seems to be dependent on the interstimulus interval (Ances et al 2000a): CBF response is less important when interstimulus time is short, while somatosensory EP's response is not altered, suggesting that hemodynamic response but not neuronal response is refractory (Figure 2). The spatial extent of activation in functional magnetic resonance imaging (fMRI) experiments has been found to correlate to a reasonable extent with NA measured neurophysiologically, considering the differences between these IT (Detre et al 2002).

Glutamate might not be the only neurotransmitter that is involved in NVC via glutamate transporters, an increased $\mathrm{Na}^{+}$followed by activation of a specific glial $\mathrm{Na}^{+} / \mathrm{K}^{+}$-ATPase isozyme (Cholet et al 2002). Acetylcholine (ACh) that is produced by the basal forebrain neurons increases CBF in specific cortical (mainly frontoparietal) and subcortical areas (Atochin et al 2003). This subcellular effect might be due to the direct contact of basal forebrain neurons or due to the presence of cholinergic receptors on nitric oxide synthetase (NOS)-containing neurons (Atochin et al 2003) and underlies a physiological age-related impairment of NVC (Hock et al 1995). The neuronal system also plays an important role in the coupling mechanism between NA and functional CBF response (Tsukada et al 2000) being attributable to the alterations of mechanisms of coupling via the cholinergic system not only in $\mathrm{AD}$, but also in physiological aging (Meyer et al 2002). Although the exact (sub)cellular mechanisms are unclear, pathological depletion of Ach is therefore also encountered in vascular dementia. It seems that vascular lesions damage cholinergic neurons (Meyer et al 2002).

\section{The coupling between cerebral blood flow and cerebral metabolism}

The hypothesis of NVC is proposed initially by Roy and Sherrington in 1890 (cited in Weller et al 2002) that activity-induced increase in glycolysis mediates the increase in CBF by mechanisms linked through the near-equilibrium

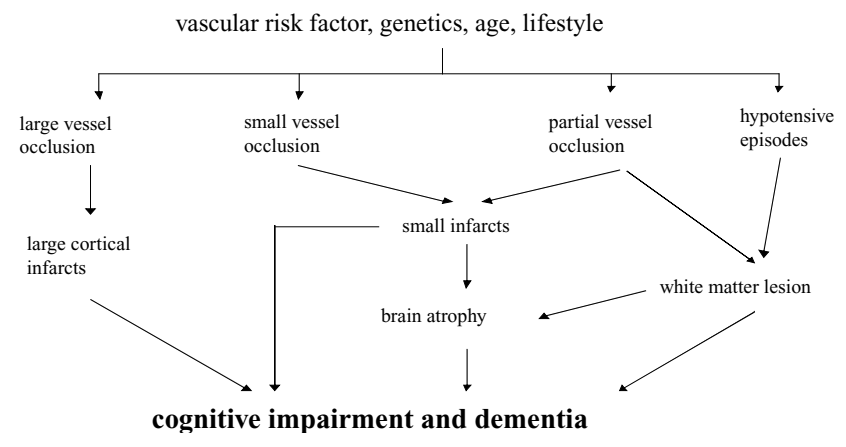

Figure 2 Relationship between hemodynamic parameters and dementia syndromes. 
relationsship between cytosolic NADH/NAD+ and the lactate/pyravat ratios (Wolf et al 1997). There is evidence that the intracellular NAD+/NADH and the related extracellular ratio of lactate to pyruvate play an importance as products of cerebral metabolism as well as chemical stimuli are key factors that determine CBF rate (Wolf et al 1997; Ames III 2000). CBF in the activated brain is regulated by the need to support very specific and vital reactions such as glutamte uptake and cycling by astrocytes (Wolf et al 1997). $\mathrm{CBF}$, however, may increase without significant change in local metabolism, and may increase much faster than the accumulation of the metabolic end-products (Magistretti et al 1999). The regulation of coupling should thus involve physiological mechanisms that are independent of energetic substrates (Magistretti 2000). CBF changes in the physiologically stimulated brain is thus related to changes in glyclolysis predominantly in astrocytes and is aimed to balance the cytosolic NADH/NAD+ ratio (Wolf et al 1997). Neurogenic stimulation via perivascular nerve endings may act as a rapid initiator, to induce a moment-to-moment dynamic CBF adjustment to the metabolic demands, and further maintenance of these adjusted parameters is ensured by metabolic and chemical factors (Sandor 1999; Zonta et al 2003). Constrictions appears spatially restricted, maximal at the level of neurite apposition, and is associated with contraction of surronding smooth muscle cells, providing the first evidence for neuronal regulation of vascular sphincters (Riddle et al 2003; Cauli et al 2004). Vascular cells are functionally linked to the neural tissue (Zheng et al 1991), making them essential members of the diverse family of brain cells that together constitute the neurovascular unit: Perivascular nerve endings were identified in the outer smooth muscle layer of the cerebral arteries, arterioles and veins (Sandor 1999). Their axon terminals contain a large variety of neurotransmitters, often co-localized in synaptic vesicles (Sandor 1999). Stimulation of these nerves results in a release of transmitters into narrow neuromuscular synaptic clefts of the cerebrovascular smooth muscles, close to specific receptor sites in the vessel wall (Sandor 1999). Several vasoactive molecules have been implicated in two types of regulation: (i) a local metabolic regulation exerted by NO, arachidonic acid derivates and adenosine or (ii) a loco-regional neurogenic regulation exerted by classical neurotransmitters and peptidergic neuromodulators (Roland et al 1987). Based on the fact that vascular responses to NA are extremely fast, neurogenic regulation and $\mathrm{NO}$ appears to represent major candidates for a role in the dynamic adaptation of $\mathrm{CBF}$ (Ogawa et al 1996). This context is elegantly illustrated in that NA causes vasodilatation through the ability of specific subsets of NO-producing GABA interneurons to transmute neuronal signals into vascular responses and further suggest that they could act as local integrators of NVC (Ranicillac et al 1996; Cauli 2004; Zlokovic 2005).

Highly regulated metabolic interactions occur between neurons and astrocytes, providing a conceptual framework for the interpretation of new experimental data on the molecular aspects of brain energy metabolism (Magistretti 2000). Through the use of autoradiographic techniques in animalmodel's brain activity at rest, a linear relationship between $\mathrm{CBF}$ and $\mathrm{CMR}_{\text {glc }}$ has been demonstrated (Zlokovic et al 2004). A quite similar correlation has been found between $\mathrm{CBF}$ and $\mathrm{CMR}_{\mathrm{O} 2}$ at the resting state (Roland et al 1987). Such a relationship between CBF and neuronal metabolism, so-called "flow-/metabolism-coupling," is observed whatever technique or species is used (Gsell et al 2000; Snyder et al 2005). Although the existence of a flow-/metabolismcoupling at rest is well accepted, the parallelism between $\mathrm{CBF}$ and cerebral metabolism appears to be far from clear during NA paradigms. There is considerable heterogeneity, for example, in the density of brain capillaries and in the distribution of mitochondria responsible for aerobic metabolism (Nag 2003). Coupling between $\mathrm{CBF}$ and $\mathrm{CMR}_{\mathrm{glc}}$ is quite well preserved in a vast majority of cerebral regions that are activated following either mechanical or electrical stimulation under experimental conditions, but several uncoupled situations have been observed in subcortical regions such as the thalamus, a brain region in which the CBF response is found to largely exceed that one of $\mathrm{CMR}_{\mathrm{glc}}$ under physiological conditions (Ueki et al 1988; Buxton et al 2000; Gsell et al 2005). Hemodynamic responses correlate better with local mean field EP, rather then local spiking rates, suggesting that CVC is dominantly driven by input synaptic activity rather than output spiking activity (Lauritzen et al 2003). The primary expenditure of energy is required to restore the ion gradients degraded during NA (Magistretti et al 1999; Buxton et al 2004). The intracellular - extracellular $\mathrm{Na}^{+}$-gradient is far from equilibrium, so pumping $\mathrm{Na}^{+}$against this gradient represents a strongly uphill reaction in a thermodynamic sense (Buxton et al 2004). For this reason, the most costly aspect of NA is likely to be excitatory synaptic activity in which glutamate opens $\mathrm{Na}^{+}$channels (Magistretti 2000; Buxton et al 2004). Indeed, the action of the $\mathrm{Na}^{+} / \mathrm{K}^{+}$pump is thought to consume a large fraction of the ATP energy budget in the brain (Magistretti et al 1999; Ames III 2000). The maintenance of such an energy state despite constant metabolic rate in the somatosensory cortex suggests that the 
generation of EP does not require measurable amounts of energy under pathological conditions, like in postischemia or poststroke conditions (Ueki et al 1988). Several technical points have been proposed to explain such pathological situations of uncoupling between $\mathrm{CBF}$ and $\mathrm{CMR}_{\text {glc }}$ under experimental conditions and to exclude nonpathological conditions: (i) a difference of the temporal resolution between imaging techniques for $\mathrm{CBF}$ versus $\mathrm{CMR}_{\text {glc }}$; (ii) a low spatial resolution not allowing the detection of very small regions of interest; and (iii) a different size of the activation foci for $\mathrm{CBF}$ and cerebral metabolism. For this latter point, a larger activation is needed in comparison to a region delineated by the $\mathrm{CMR}_{\mathrm{glc}}$ changes; this can also result in misleading interpretations (Howard et al 1998). Therefore, the hypothesis of a nonlinear coupling between $\mathrm{CBF}$ and $\mathrm{CMR}_{02}$ has been proposed (Magistretti et al 1999): With increased NA, CBF increases substantially, while $\mathrm{CMR}_{02}$ increases only moderately, so the oxygen extraction fraction (OEF) is reduced (Forette et al 1998). In addition, a nonunit gain relationship is actually expected due to the diffusion limitation of oxygen supply. However, astrocytic lactate can replace glucose as an energetic supply for neurons during activation has suggested that, at least, part of the glucidic metabolism could be anaerobic recycling NAD+ via the reduction of pyruvate to lactate despite the presence of sufficient oxygen and export the lactate (Pantel et al 1998). It seems that not all carbohydrate taken up by the brain is oxidized during activation, as brain lactate metabolism appears to lower the balance of $\mathrm{O}_{2}$-tocarbohydrate uptake (Ide et al 2000). Lactate seems then to be imported into the neuron and to feed oxidative metabolism in neurons (Ide et al 2000). Under these conditions one can hypothesize nonparallel variations of $\mathrm{CMR}_{02}$ and $\mathrm{CMR}_{\mathrm{glc}^{\circ}}$ If $\mathrm{CBF}$ is a robust index of NA, it might be tightly coupled to both, metabolism and NA, and might thus not vary alone while the two other parameters are stable (Magistretti 2000; Schaller et al 2004b). However, experimental data suggest a situation of uncoupling between $\mathrm{CBF}$ and, at least, metabolism under pharmacologically induced conditions indicating that the involvement of neuron-derived nitric oxide (NO) in coupling flow to somatosensory activation is region-dependent, and that under nNOS inhibition, $\mathrm{CBF}$ and $\mathrm{CMR}_{\text {glc }}$ may vary independently during NA (Dauphin et al 1995); therefore lactate, rather than glucose, may be the preferred fuel for neuronal metabolism (Slosman et al 2001). However, the large carbohydrate uptake by the brain during recovery from maximal activation suggests that brain glycogen metabolism is important in the transition form rest to exercise, since this would explain the significant post- activity decrease in the $\mathrm{O}_{2}$ /carbohydrate uptake ratio (Ide et al 1999). Therefore, circulating lactate is used by the brain at euglycemia, with sparing glucose (Slosman et al 2001).

\section{The neurogenic regulation of coupling by acetylcholine}

Ach is a potent vasodilator innervating local cortical nitroxidergic interneurons, cerebral capillaries and microarterioles (Herholz et al 2004). Cholinergic innervation mostly originates from the nucleus basalis of Meynert (Mesulam et al 1983). The existence of such innervations, associated with the presence of cerebrovascular muscarinic receptors, has allowed to propose the implication of an ascending cholinergic system in the regulation of cortical CBF (Dauphin et al 1995). If cholinergic innervation is necessary to maintain $\mathrm{CBF}$ control in specific brain regions, degeneration of basal forebrain cholinergic neurons, as in $\mathrm{AD}$, could therefore uncouple NA from CBF (or alter the nature of the coupling) locally as well as in specific remote regions (Honer et al 1988; Blin et al 1994). Altered CBF response in such specific brain regions could not directly be interpreted as altered NA (Herholz et al 2004; Rosengarten et al 2006). One may hypothesize for these reasons that up-regulation of eNOS directly augments the neuron to vascular communication or that the increased levels of vasorelaxing NO only improve the endothelial site of the coupling resulting in a better responsiveness (Rosengarten et al 2006). The clinical finding of pharmacological effect of acetylcholine esterase (AchE) inhibition in patients suffering from a vascular related dementia may support the latter notion (Pratt et al 2002). Moreover, the nonuniform cholinergic cortical innervation could explain the regional variability in NVC in certain circumstances (Herholz et al 2004).

AchE inhibitor physiostigmine has been found to increase basal $\mathrm{CBF}$ and to decrease $\mathrm{CMR}_{\text {glc }}$, leaving $\mathrm{CMR}_{\mathrm{O} 2}$ unchanged (Schaller 2005). Conversely, decreased CBF and increased $\mathrm{CMR}_{\mathrm{glc}}$, a phenomenon that also illustrates a situation of uncoupling, follows systemic injection of the muscarinic antagonist scopolamine to humans or animals (Hof and Morrison 2004). Seigneur and colleagues (2006) suggest that the response to cholinergic activation may therefore result from the balance between the direct hyperpolarizing action of ACh and the depolarizing modulation of glutamate from the neighboring neurons, in addition to the modulation of the interglial communication pathway and/or the ionic traffic across blood vessels. In addition, during somatosensory activation, increased $\mathrm{CBF}$ has been found to be abolished by pharmacological intervention (eg, by scopolamine or by 
bromopyruvate) such as inhibitors of ACh synthesis, and to be restored by physiostigmine (Tsukada et al 2000). Again, the neuronal response under pharmacological manipulation in these experimental studies is not measured through electrophysiological recordings but assumed by the preservation of the metabolic response (Tsukada et al 2000). Therefore, one cannot conclude on an uncoupling between $\mathrm{CBF}$ and NA but only between $\mathrm{CBF}$ and cerebral metabolism under these conditions until all three parameters have been measured.

AchE activity in cerebral cortex is mainly due to the expression of this enzyme in cholinergic neurons and their axons (Herholz et al 2000). Intrinsic cholinergic neurons of the basal cortical pathway participate physiologically in the regulation of CBF responses (Tsukada et al 2000). Indeed, the direct stimulation of the substantia innominata or the nucleus basalis of Meynert elicits an increased cortical CBF that is not associated to a parallel change in $\mathrm{CMR}_{\text {glc }}$ (Vaucher et al 1997) being hypothesized to be implicated in the brain parenchyma during NA, suggesting that AchE modulates glial activation and CBF. In addition, nucleus basalis of Meynert lesion studies in animal-models demonstrated to decrease cortical CBF (Peruzzi et al 1993). In contrast, it has been shown that lesions of the nucleus basalis of Meynert do result in an unexpected preservation of $\mathrm{CMR}_{\mathrm{glc}}$ associated to an increase in the density of GLUT3 (glucose transporter 3) glucose transporters in the cortex (Simpson et al 1994). This could be of great importance in pathological situations, where the cholinergic system is supposed to be degenerated as, for example, in degenerative dementia with consecutive loss of cholinergic neurons located in basal ganglia, particularly in the nucleus basalis of Meynert (Herholz et al 2000). As the ascending cholinergic system appears to be involved in the neurogenic regulation of $\mathrm{CBF}$ and/or $\mathrm{CMR}_{\text {glc }}$, interestingly, ACh has been demonstrated to interact also with a local, nitroxidergic, and metabolic regulation (Herholz et al 2000).

\section{The local regulation of coupling by nitric oxide}

NO, first discovered as the "endothelial-derived-relaxingfactor", is supposed to be implicated both in the regulation of neuronal activity, $\mathrm{CBF}$, and metabolic adjustments (Furchgott et al 1980). NO is synthesized by three isoforms of NOS and has been localized next to the cerebral vessels, within nervous fibers suggesting a direct effect of NO on the CVsystem (Baumach et al 2004) and nNOS inhibitors allow demonstrating reductions in resting $\mathrm{CBF}$ as well as in $\mathrm{CBF}$ responses to somatosensory activation (Lindauer et al 1996).
Moreover, $\mathrm{nNOS}$ inhibitors failed to modify either the $\mathrm{CMR}_{\mathrm{glc}}$ response or NA as assessed by somatosensory EP (Cruz et al 2002) supporting the existence of independent mechanism for the regulation of CBF and cerebral metabolism (Lindauer et al 1996). Although NO participation in NVC is widely accepted, its exact mechanism of action remains yet unclear (Smith et al 2003). Since NO serves as a retrograde messenger in synapses, one source of NO could be a leakage from synaptic clefts and rapid diffusion from active synapses to arterioles (Longstreth et al 1996). As capillaries are much closer to pyramidal neurons than arterioles, diffusion might be adequate to provide the pyramidal neurons with nutrients from capillaries, but ineffective in delivering metabolic messages to the CBF gate at the terminal arteriole (Schaller et al 2004b) (Figure 3). Alternatively, NO could be produced by specialized periarteriolar neurons that are activated by direct stimulation from collateral axons, or by leaked neurotransmitters form active synapses (Waldmann et al 2003). In support of this notion, NOS containing neurons are located close to pyramidal cells and vary in density consistent with the variation of microvasculature density (Togo et al 2004). In addition, a network of fine fibers and staining for NOS is found around arterioles, suggesting that NOS-containing terminals provide a link between active pyramidal cells and relatively remote terminal arterioles (Togo et al 2004). NO is continuously released by endothelial cells into the vascular system; but advanced age followed by the presence of vascular risk factor causes a decreased $\mathrm{CBF}$, involving microvasculopathy with impaired NO release, which in turn results in regional metabolic dysfunction (Togo et al 2004).

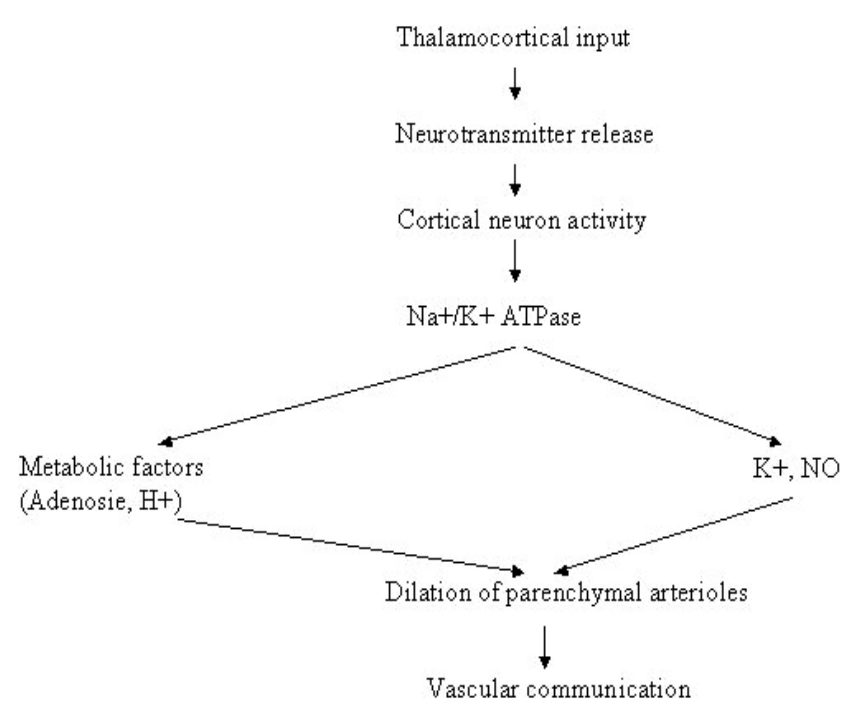

Figure 3 Neurovascular coupling mechanisms during cerebral somatosensory activity. 
Whether the control of CBF by NO is implemented through simple diffusion or through specialized networks (neuronal or glial) should determine the degree to which the activity of clusters of neurons will produce similar amplitudes and temporal profiles of CBF changes (Atochin et al 2003) that can be visualized in FIT (Jagust 2005). Another factor in this complex equation is the nonuniform distribution of NO-producing neurons in different cortical areas (see for example Schaller 2004a). However, these findings suggest that vascular pathology plays a crucial role in the pathogenesis of so-called neurodegenerative dementias (Zlokovic 2005). Inflammatory responses are commonly found in the brain under a variety of neurodegenerative dementias, including $\mathrm{AD}$ and DLB, in which up-regulation of NOS expression, suggesting overproduction of $\mathrm{NO}$, is found in neurons and glia (Togo et al 2004). NO is thought to be involved in such neuroinflammation due to its free radical qualities, which compromise cellular integrity and viability via mitochondrial damage (Togo et al 2004; Schaller 2007; Schaller et al 2007).

It is also not known whether NO is a direct mediator of enhanced $\mathrm{CBF}$ or has only a modulatory ("permissive") role. It remains unclear whether NO determines the vascular response quantitatively, or it is necessary for other mediators to exert their effect (Lindauer et al 1996). In the rat cerebellum, decreased CBF response leads to inhibition of NO indiscriminately, indicating that NO acts as a direct mediator of local CBF control (Lin et al 2005). In contrast, in the rat somatosensory cortex NO has a modulatory effect, since the application of NO restored the CBF response after inhibition of NOS (Cholet et al 1997) suggesting that NVC processes might vary across the brain.

Although no study has addressed the effect of nNOS inhibitors on the physiological parameters of interest in the same model, all these data taken together suggest dissociation between vascular and metabolic/electrophysiological responses under nNOS inhibition (Gotoh et al 2001) as underlined by the use of knockout mice lacking the genes for either eNOS or nNOS (Ayata et al 1996; Browne et al 1999). Pharmacological manipulation of NO through the blockade of its synthesis allows demonstrating regionspecific-effects; indeed, while cortical CBF response to cerebral nNOS inhibitors decreases activation, the vascular response is not altered in the thalamus (Cholet et al 1997) suggesting the existence of a complex mechanism in which the involvement of $\mathrm{NO}$ in coupling differs form one region to another.

\section{Altered cerebrovascular dynamics in physiological aging}

The vascular pathology seen in physiological aging process can be clinically silent or can lead to vascular compromise resulting in cerebral ischemia or even stroke representing probably the basis for alterations in NVC (Hock et al 1995; Terborg 2000). However, cerebrovascular risk factors may cause asymptomatic brain injury, strengthen the genetic risk with $\mathrm{AD}$ and possibly accelerate neurodegeneration-related processes (Decarli et al 2004) suggesting that these two diseases may act cumulatively or synergistically to cause clinical dementia (Decarli et al 2004).

\section{Ultrastructure of the cerebral vasculature}

The compromise to the ultrastructural integrity of the cerebral vasculature in physiological aging process is mainly the result of arteriosclerotic changes, fibrohyaline thickening of the vessel wall, necrosis of smooth muscle cells and thickening of the basement membrane, that gradually increase with age (Masawa et al 1994; Hock et al 1995). Although sclerotic changes correlate with the degree of hypertension, age seems to be an independent risk factor (Masawa et al 1994). These morphological changes decrease the elasticity and compliancy of affected vessels, which include capillaries, larger arterioles and cerebral arteries (Kacem et al 1998). Venous alterations that accompany aging, known as periventricular venous collagenosis, are also found in about $65 \%$ of the population over 60 years old, and can occlude veins completely in severe cases (Moody et al 1997). There is also an increase in the tortuosity of some vessels with physiological aging, most notably in the arteriovenouscapillary bed, as well as changes in the density of capillaries and arterioles, but this effect have not yet been observed in venules (Hock et al 1995).

\section{Resting cerebral blood flow}

Aging is associated with a rarefaction of the microvasculature followed by a significant decrease in resting CBF in the cortical and subcortical parenchyma reducing metabolic support for neuronal signaling particularly when levels of neuronal activity are high (De la Torre et al 1992; Postiglione et al 1993; Reiman et al 2004). Using positron emission tomography (PET), $\mathrm{CMR}_{\mathrm{O} 2}$ demonstrates a significant decline during physiological aging process predominantly at the left superior temporal cortex and more marked in the association cortices of the left hemisphere (Postiglione et al 1993; Reiman et al 2004). Evidence that age-related declines in $\mathrm{CBF}$ are not specific for neuronal function is 
provided by the demonstration that aging decreases both basal CBF and the increase of CBF normally accompanying neuronal stimulation (Reiman et al 2004). However, considering the significance of age-related changes in $\mathrm{CBF}$ and/or metabolism, two caveats exist: (i) aging-related cerebral atrophy may confound the interpretation of imaging studies of CBF (Giovacchini et al 2004); and (ii) a decreased local $\mathrm{CBF}$ in the aged brain could be a secondary rather than a primary factor of a decline in neural function, especially in the grey matter (Illes et al 2004). However, there has been discussion as to whether neuronal loss subtending gross structural changes in aged healthy volunteers fully explains the regional changes measured with functional imaging (van Laere et al 2001). Contrary to the widely belief, neuronal concentration remains essentially constant during normal aging process, but neuron size decreases - presumably as a result of reduced dendritic arborization and dendritic spine concentration (Anderson et al 1996) - and the number of glial cells decreases (Baron et al 2000) occuring with substantial variability (van Laere et al 2001).

\section{Vascular reactivity}

Specific, age-associated decrease in the vascular reactivity of cerebral vessels to various chemical modulators, including carbon dioxide $\left(\mathrm{CO}_{2}\right)$ is seen (Bakker et al 2004). Generally accepted, increased blood $\mathrm{CO}_{2}$ results in dilatation of cerebral arterioles under physiological conditions (Venton et al 2003). Decreased vascular reactivity to hypercapnia has been observed in aged animals or humans with and without risk factors for arteriosclerosis (Wolfson et al 1985). In PET of $\mathrm{rCBF}$, a significant decrease in a total vascular response from a hypocapnic to a hypercapnic state is observed in older individuals as compared to younger ones (Mufson et al 2003; Reiman et al 2004). In aged animals, there is a reduced degree of vasodilatation in response to cerebrospinal fluid perfusion of the vasodilators adenosine, ACh and bradykinin (Mayahn et al 1990).

The exact mechanism of age-related changes in vascular reactivity have not been elucidated, although it has often been suggested that aging-related effects may be most significant for agents that act by modulating the release of endotheliumderived relaxing factor (NO) (Riecker et al 2003; Rodrigo et al 2004; Ranicillac et al 2006). But, the wall of the cerebral arterioles undergoes atrophy during aging and there is a decrease in the distensibility of the cerebral vessels, which is mainly based on alterations of their composition with a disproportionate reduction of the distensible elements (Hajdu et al 1990). Therefore, cerebral vessels in older subjects may not react as effectively in response to dilator stimuli such as hypercapnia or those released during neuronal activation (Riecker et al 2003). In addition to such structural changes, mechanisms that might affect vascular reactivity independent from NA include the gliosis that accompanies tissue scarring from damaged brain tissue, and the disruption of long-range aminergic and cholinergic fibers that innervate the vasculature (Czosnyka et al 2004). Thus, altered NVC can occur close to a damaged brain region even in apparently normal tissue (Back et al 1998). Moreover, age-related changes in vascular reactivity might vary across brain regions (HundGeorgiadis et al 2003). A study that compared the resting and stimulus-evoked increases in CBF ipsilateral in both the parietal and frontal cortex of young rats demonstrated altered effects in the frontal cortex of aged rats (Ranicillac et al 2003). This finding suggests that the structural and functional changes that occur in vessels during physiological aging cause disability of cerebral microvessels to deliver nutrients and oxygen to the brain optimally, thereby affecting the mitochondrial ability to respond to anoxia (Mufson et al 2003) mirroring the nonuniform distribution of arteriosclerosis in the brain (see for example Paul et al 2005).

There is an age-related dissociation of calbindin and the glutamate receptor GluR2 within cholinergic basal forebrain neurons, implying that these molecules act synergistically to induce excitotoxic cell death during aging, and possibly during AD (Mufson et al 2003). Choline acetyltransferase (ChAT) activity and cholinergic basal forebrain neuron number are preserved in the cholinergic basocortical system and up-regulated in the septohippocampal system during early as compared with end-stage AD (Mufson et al 2003). In contrast, the number of cholinergic basal forebrain neurons containing nerve growth factor (NGF) receptors is reduced early in the disease suggesting a phenotypic silence and not a frank loss of neurons (Mufson et al 2003). In end-stage $\mathrm{AD}$, there is a selective reduction in trkA mRNA but not p75(NTR) in single cholinergic basal forebrain cells suggesting a neurotrophic defect throughout the progression of AD (Mufson et al 2003; Zlokovic 2005) and indicating that multiple factors may play different roles in the consecutive dysfunction in aging and AD (Mufson et al 2003).

\section{Additional significance of aging-related changes in vascular plasticity}

Aging-related changes in the vasculature also influence other critical aspects of neural function and plasticity (Mark et al 1997). The microvasculature is critically involved in regulating adult neurogenesis, as a source of local and blood-borne 
factors providing an appropriate environment for neurogenesis stimulating the proliferation and differentiation of progenitor cells (Schaller 2004a). For this reason, the role in brain repair after ischemic events is under discussion: The production of new granule neurons in the subgranular zone of the adult dentate gyrus occurs within "neuroangiogenic foci" where neuronal, glial and endothelial precursors are divided into tight clusters (Schaller 2004a; Togo et al 2004). This association between endothelial and neuronal proliferation in the subgranular zone suggests that signals originating from somatic tissues or from the CNS act simultaneously to stimulate neurogenesis and angiogenesis, or that the initiating signal activates proliferation of only one cell type, which then stimulates proliferation of the other (Schaller 2004a). In addition to trophic factors, endocrine growth factors like insulin-like growth factor (IGF-1) influence neuronal turnover in the adult brain, regulating neurogenesis, and the decline in neuronal turnover during advanced aging may be the result of decreased IGF-1 levels (Sokoloff, 1981).

\section{Pathological-induced alterations of cerebral blood flow and/or metabolism regulation Cerebral blood flow}

The collective findings discussed so far suggest that brain hypoperfusion precedes the hypometabolic and neurodegenerative changes (Ruitenberg et al 2005), investigating early stages of dementia in which advanced neurodegeneration have not yet evolved. In the opposite sequence, neurons exposed to oxidative stress and impaired energy substrate delivery would further reduce $\mathrm{CBF}$ to accelerate neuronal death, a mechanism inconsistent with cellular survival (Zlokovic 2005). The use of cerebral metabolism as an index of NA may not always be valid, particularly when the biochemical steps related to cellular energy and cellmembrane turnover is altered like in AD (Silverman et al 2005). Therefore adequate measurement methods of NA would be needed to examine the preservation or alteration of NVC in these pathological states: in these selected situations, CBF variations appear to be non reliable indices of cerebral metabolism and, putatively, of neuronal activity (Gsell et al 2000). This may limit the use of activation IT in pathophysiological conditions (Magistretti et al 1999). Another major problem is represented by the yet unknown coupling between oxidative metabolism and CBF: Techniques such as fMRI and near infrared spectroscopy (NIRS), that record a complex signal linked both to variations of $\mathrm{CMR}_{02}$ and $\mathrm{CBF}$, might thus fail to distinguish between these two parameters (Gesell et al 2000).

Specific pattern of reduced $\mathrm{CBF}$ and metabolism have been demonstrated for several subtypes of dementia (Silvermann et al 2004). For a number of authors, decrease of $\mathrm{CBF}$ and glycolytic metabolism are tightly correlated to the severity of the disease (Jobst et al 1992; Pietrini et al 2000). Alterations of CBF and cerebral metabolism have been presented over the years as parallel events that should directly reflect the local loss of neurons and, in turn, the decrease in the local metabolic demand (Frachowiak et al 1981). However, uncoupled variations of resting CBF and metabolism exist in AD (Nagata et al 1997). In fact, a slight increase in $\mathrm{CMR}_{\mathrm{O} 2}$, suggesting a so called "misery perfusion", has been found in some brain regions of AD (Nagata et al 1997). Moreover, behavioral activation and $\mathrm{CMR}_{\mathrm{glc}}$ measurements by PET suggest that the regional hypometabolism found in $\mathrm{AD}$ is not the result of neurodegeneration, but appears to precede it (De la Torre et al 2002).

Though neuronal loss alone cannot explain the cerebrovascular and metabolic alterations observed in $\mathrm{AD}$, the pathophysiological mechanisms, which alter CBF and/or local metabolism, are still unknown (Nixa et al 2002; Iadecola et al 2003), but it seems that this phenomenon may be an early event in the pathogenesis of AD. Different working hypotheses have recently been suggested, stressing the importance of alterations of the ascending cholinergic pathway and of endothelial functions as well as of pathophysiological perturbations associated with $\beta$-amyloid protein (AP) overexpression (Poirier et al 2000). It is thus quite tempting to propose that two of the most common neuropathologic landmarks of $\mathrm{AD}$, namely, cholinergic dysfunction and $\mathrm{AP}$ deposition, may in fact depend on the integrity of local lipid homeostatic processes, which in turn are strongly dependent upon proper lipid delivery by the apoE transport system (Poirier et al 2000).

The argument that hypometabolism in $\mathrm{AD}$ may elicit microvascular changes at some point is not supported by a considerable number of animal experiments. But chronic cerebral hypoperfusion can trigger oxidative stress, energy metabolic deficits, and memory loss before any neuronal structural pathology materializes (Chen et al 2005; Sun et al 2005), whereas there are no data that we are aware of that indicate the reverse process can or does occur. Moreover, the recent discovery of "neuroglobin" in rodent and human brain could partly explain why CA1 hippocampal neurons are exquisitely sensitive to hypoperfusion leading 
to hypometabolism (Chen et al 2005; Sun et al 2005). Neuroglobin in brain appears to act much like myoglobin in cardiac muscle cells; it aids oxygen diffusion to the mitochondria (Chen et al 2005). Decreased tolerance of CA1 to ischemia may be due to lower oxygen supply resulting from less available neuroglobin, whose lowest expression is in the hippocampus (Chen et al 2005). Consequently, brain hypoperfusion could reduce neuroglobin levels in CA1 and initiate mitochondrial oxidative stress (Chen et al 2005).

\section{Cholinergic pathways}

Cholinergic pathways projecting from basal forebrain regions to the hippocampus and neocortex are believed to play an important role in learning and memory processes (Bartus et al 1982). In patients with AD, the cholinergic system of the basal forebrain, including the areas of projection, is disrupted and/or altered (Collerton 1986). Moreover, as direct stimulation of the nucleus basalis of Meynert induces a major increase in CBF in the cortex without variation of the glucose metabolism, thus suggesting a preferential regulation of CBF by this region (Barbelivien et al 1999). Interestingly, a reduction in the density of the muscarinic receptors within cerebrovascular bed has been reported in $\mathrm{AD}$, a situation that, associated to the parallel decrease in the density of contacts between cholinergic terminals and nitroxidergic cortical interneurons, could be responsible for an uncoupled state between CBF and cerebral metabolism (German et al 2003). Such a situation might be exacerbated by the alterations of the modulation of the cholinergic system of the nucleus basalis of Meynert, for example by galanin or GABA, known to modify the functional input of the cholingeric system onto CBF regulation (Barbelivien et al 1999). These data indicate that the cortical neurodegenerative changes occur before the deposition of AP (German et al 2003).

\section{Glucose pathways}

Glycolytic enzymes and glucose transporters present at the level of the blood-brain barrier (BBB) and neurons also appear to exhibit an abnormal function during AD (Kalaria et al 1989; Mielke et al 1996). In particular, a decrease in the density of GLUT1 and GLUT3 glucose transporters has been reported (Simpson et al 1994). Overall, these alterations should induce metabolic changes characterized by an important decrease in $\mathrm{CMR}_{\mathrm{glc}}$; since $\mathrm{CMR}_{\mathrm{O} 2}$ is supposed to be less decreased, this should elicit an increase in the $\mathrm{CMR}_{\mathrm{O} 2} / \mathrm{CMR}_{\mathrm{glc}}$ ratio, characteristic of a shift towards aerobic metabolism (Fukuyama et al 1996).

\section{Amyloid- $\beta$}

A number of in vivo neuroimaging techniques, which can reliably and noninvasively assess aspects of neuroanatomy, chemistry, physiology, and pathology, hold promise as biomarkers (Dickerson et al 2005): Apolipoprotein E4 (apo E4) is a component of both the AP and the tangles of hyperphosphorylated tau that characterize AD (Mullan et al 1993). Furthermore, in AD early accumulation of AP in several brain regions as well as in the vascular compartments has been demonstrated (Mullan et al 1993). Amyloid angiopathy induces a structural alteration of the vessel wall, an endothelium-dependent perturbation of the cerebrovascular reactivity and an increase in the BBB permeability (Blanc et al 1997) possibly leading to hemorrhage and ischemia (Koistinaho et al 2005). Moreover, intra-arteriorlar injection of AP reduces the internal diameter of vessels and thereby can decrease $\mathrm{CBF}$ and/or energetic supply to the cerebral parenchyma (Duara et al 1992). Accordingly, several studies of cerebral ischemia have shown similar alterations. Indeed, chronic hypoperfusion in the rat has been found to induce alterations of brain capillaries associated with an overexpression of the AP precursor, changes in the function of glucose transporters, and dysfunction of the cholinergic system (Ishimaru et al 1998). Currently, different tools that enable direct, quantitative detection of AP deposits found in $\mathrm{AD}$ support the existence of an excellent biomarker for different imaging modalities and are part of current experimental research (Dickerson et al 2005).

\section{Nitric oxide pathways}

NOS is supposed to be the pivotal factor in the pathogenesis of different subtypes of dementia, especially AD (Lahiri et al 2003; Rodrigo et al 2004) demonstrating (i) the release of NO from cerebral endothelial cells to produce vasodilatation, a fairly well established reaction (Iadecola et al 2003); (ii) NO produced by eNOS to play a key role in vascular tone and blood pressure control, and to inhibit platelet and leukocyte adhesion to the endothelium, a process that may down-regulate proinflammatory events (Iadecola et al 2003); (iii) the stimulation angiogenesis via vascular endothelial growth factor reduces shear stress on the vessel wall and, consequently, protects endothelial cell function (Lahiri et al 2003). NOS dysfunction may therefore disrupt the delicate balance between the brain's energy requirements and blood supply, and render the brain more vulnerable to (ischemic) injury (Iadecola et al 2003).

NO had been shown to diffuse toward the lumen of blood vessels where it helps maintain $\mathrm{CBF}$, by reducing blood 
viscosity and resistance (Niwa et al 2002a). However, normal blood flow can be compromised through physical or chemical stimuli affecting eNOS levels localized in the endothelial cell caveolae (small invaginations in the plasma membrane of endothelial cells involved in cell signalling) (Hof et al 2004; Iadecola 2004). Thus, deregulation of vascular NO production can occur from chronic cerebral hypoperfusion (Niwa et al 2002a, 2002b) as follows: chronic cerebral hypoperfusion below a critically attained threshold initiates eNOS activation and deregulation of NO release from the endothelium in the hippocampus and endorhinal cortex, results in an increased vascular resistance or blood viscosity and causes micro-environmental hemorheological and hemodynamical disturbances (Niwa et al 2002b). This effect of altered mechanotransduction on eNOS activity up-regulates NO release in order to reset baseline values and diminish the molecular and cellular damage caused by hypoperfusion (Niwa et al 2002b; Iadecola 2004). In view of failure to achieve homeostasis, depletion of basal NO levels results in the incapability (i) to regulate normal vascular perfusion, (ii) to block granulocyte adhesion in blood vessels, (iii) to prevent proinflammatory intraneuronal reactions, and (iv) to maintain endothelial cell conformation, resulting in endothelial cell shape distortions (Iadecola 2003). The latter phenomenon may explain basement membrane thickening commonly found regionally in $\mathrm{AD}$ brain capillaries and potentially seen as a compensatory reaction to increased interendothelial distance created by $\mathrm{NO}$ deregulation (Niwa et al 2002b). Endothelial cell distortion would also permit mobilization of the powerful vasoconstrictor, endothelin-1, and impair GUT-1 activity, the molecule responsible for glucose transport into brain (Iadecola 2003). Thus, AP compromises the ability of cerebral endothelial cells to produce vascular relaxing factors, impairs the ability of cerebral blood vessels to maintain adequate flow during hypotension, and attenuates increased $\mathrm{CBF}$ evoked by enhanced NA (Iadecola 2003).

NO appears to affect nuclear factor kappa beta (NF- $\kappa \beta)$ directly, a nuclear transcription factor that is widely expressed in CNS and that is involved in the activation of several inflammatory mediators (Howard et al 1998; Schaller et al 2007). Simultaneously to NF- $\kappa \beta$ activation, in most types of cells, inhibitor I $\mathrm{IB} \alpha$ is phosphoryled and proteolytically degraded (Howard et al 1998; Camanduola et al 2000). After activation, free NF- $\kappa \beta$ dimers are translocated into the nucleus, bind to the promoter region of target genes and induce the transcription required for DNA binding and protein dimerization (Howard et al 1998). However, a TNF- $\alpha$-induced NF- $\kappa \beta$-activation mediates the expression of adhesion molecules on the endothelial surface. NO inhibits NF- $\kappa \beta$ transcriptional activation in a variety of cells, including monocytes and endothelial cells (Howard et al 1998). When NO is activated by TNF- $\alpha$, the $\mathrm{NF}-\kappa \beta$ inhibitor, inhibitor of NF-kappaBalpha $(\mathrm{I} \kappa \mathrm{B} \alpha)$, is no longer stabilized and inflammatory cytokines, such as interleukin-1 and interleukin-6, are activated in the brain (Howard et al 1998; Camanduola et al 2000). Structural microvessel pathology, which is frequently observed in $\mathrm{AD}$ brains, results in basement membrane thickening, buckling of the lumen, and distortion of the endothelium (Zhen et al 1991). Such histopathological changes will alter normal NO release from the endothelium, allowing $\mathrm{I} \kappa \mathrm{B} \alpha$ degradation and thereby preventing proinflammatory downregulation via inhibition of NF- $\kappa \beta$ (Howard et al 1998). Thus, it is possible that endovascular NO, derived from eNOS, may inhibit $\mathrm{NF}-\kappa \beta$ tonically under nonstimulated conditions (Iadecola 2004). However, during advanced physiological aging, enhanced by vascular risk factors reducing cerebral perfusion further, this mechanism may be lost, increasing the sensitivity to NF- $\kappa \beta$-activation (Howard et al 1998). In agreement with the hypothesis connecting vascular NO levels with $\mathrm{AD}$ is the finding that in the presence of NO donors, TNF- $\alpha$ fails to stimulate $\mathrm{NF}-\kappa \beta$ released from endothelial cells, allowing NO to stabilize the NF- $\kappa \beta$ inhibitor, I $\kappa \mathrm{B} \alpha$, and to prevent its degradation (Howard et al 1998). The binding of NF- $\kappa \beta$ to the $\mathrm{NF}-\kappa \beta$ binding site in the inducible NOS promoter plays a crucial role in the transcriptional regulation of the inducible NOS gene (Howard et al 1998). Furthermore, NO donors can directly inhibit the DNA binding activity of NF- $\kappa \beta$ (Howard et al 1998). Briefly, NO derived from eNOS has the ability to downregulate proinflammatory events by inhibiting $\mathrm{NF}-\kappa \beta$ activation of proinflammatory cytokines leading to microglial activation and neuronal damage (Ho et al 2005).

\section{Neurovascular hypothesis}

Neurovascular uncoupling suggests various neurovascular related pathogenic mechanisms for the origin of AD (Iadecola 2004). Faulty clearance of AP across the BBB by aberrant angiogenesis or endothelial aging process could lead to formation of vascular amyloid lesions (Figure 4) (Zheng et al 1991). Inefficient AP proteolysis amplifies insufficient AP BBB clearance, whereas AP vascular accumulations stimulate proteasome-dependent degradation of the lipoprotein receptor-related protein (LRP) clearance receptor and is antiangiogenic (Zheng et al 1991).

As suggested by Zlokovic (2005), premature cerebrovascular senescence owing to atherosclerosis, cerebral amyloid 




Figure 4 Neurovascular mechanism of Alzheimer's disease. (Zlokovic 2005). Abbreviation: BBB, blood-brain barrier.

angiopathy and/or elevated soluble AP concentration can result in loss of endothelium-dependent CBF regulation, in neurovascular uncoupling, and in brain hypoperfusion (Zheng et al 1991). Islets of regressed and degenerated microvessels consequent to aberrant angiogenesis could act as seeds for the dense-core amyloid plaques and might activate the neuroinflammatory response (Zheng et al 1991), but our understanding of the mechanisms by which vascular dysregulation alters brain function is still rudimentary.

\section{The state-of-the-art-imaging}

Biological dementia process begins years before it is diagnosed clinically (Mosconi et al 2005) (Figure 5). The optimal time to initiate a neuroprotective, therapeutic step is during the preclinical period of the disease, when neuronal degeneration has begun, but cellular and biochemical damage is not yet sufficient for symptoms to manifest (Mathis et al 2005). The ability to identify this preclinical state is currently very limited (Iadecola 2004; Chong et al 2005). The most useful strategy is to identify "at risk" subgroups and to search for preclinical neurodegeneration in these enriched populations (Zheng et al 1991). The promise of achieving diagnostic specificity through neuroimaging lies with the potential identification of pathognomonic proteins (Mathis et al 2005).

Recent advances in imaging AP, one of the hallmarks of $\mathrm{AD}$, offer such a technological breakthrough and the possibility for more efficient assessment of antiamyloid interventions as well as specific noninvasive diagnostic capabilities (Mathis et al 2005).

\section{Structural imaging}

Clinical symptoms of AD result from synaptic dysfunction that may be associated with or precedes neuronal loss, although the underlying mechanisms are unknown (Snyder et al 2005). Neuropathological studies have implicated the gray matter of the medial temporal lobe as an early site of pathological involvement in AD (consistent with the characteristic amnesic presentation in this disorder), and many IT have therefore focused on this part of the brain (Alexander et al 1995; Paris 1997). MRI enabled the visualization of specific structures within the medial temporal lobe, such as the hippocampus itself, the parahippocampal gyrus, subiculum, entorhinal cortex, and amygdale (Chong et al 2005) with good clinical correlations to dementia (Earnest et al 1954).

The future focus of high-resolution structural MRI on these areas that are affected earliest in AD (ie, entorhinal cortex and hippocampus) may increase diagnostic precision (Chong et al 2005). With the ability to acquire rapidly high-contrast, high-spatial-resolution, three-dimensional IT (Magistretti 2000; Thompson et al 2004), sophisticated brain-mapping algorithms identify complex, shifting patterns of brain structural deficits, revealing where, and at what rate, the path of brain deterioration in illness deviates from normal (Csernansky et al 2005). Results vary, depending in part on the specific measurement approaches (eg, volumetric, planimetric, linear), and many investigations suffer from limitations similar to those described for the qualitative mesiotemporal atrophy study (Poulin et al 2002). The measures of the temporal cortices, including the amygdala, hippocampus, and inferior temporal lobes, along with the anterior cingulate cortex, associated with the largest magnitudes of effects, and, hence, these could serve as the most useful structures to help clinicians differentiate AD from physiological aging (Poulin et al 2002).

\section{Cerebrovascular disease}

Silent cerebrovascular brain damage is associated with diminished cognitive performance in older people (Hock et al 1995) suggesting that CVD is associated with increased risk of mild cognitive impairment and of conversion to dementia (Ross et al 2005). In addition, the presence of CVD may influence clinical presentation and neurobiology of $\mathrm{AD}$ and seem to contribute to determine cognitive impairment in an additive fashion (Ross et al 2005), suggesting that cognitive impairment in stroke patients may be related to cortical neuronal dysfunction rather than purely subcortical change (Mackowiak-Cordoliani et al 2005). Moreover, cortical regions not obviously infarcted may have dysfunctional neurons, the pathophysiological basis for which needs further study (Ross et al 2005). 


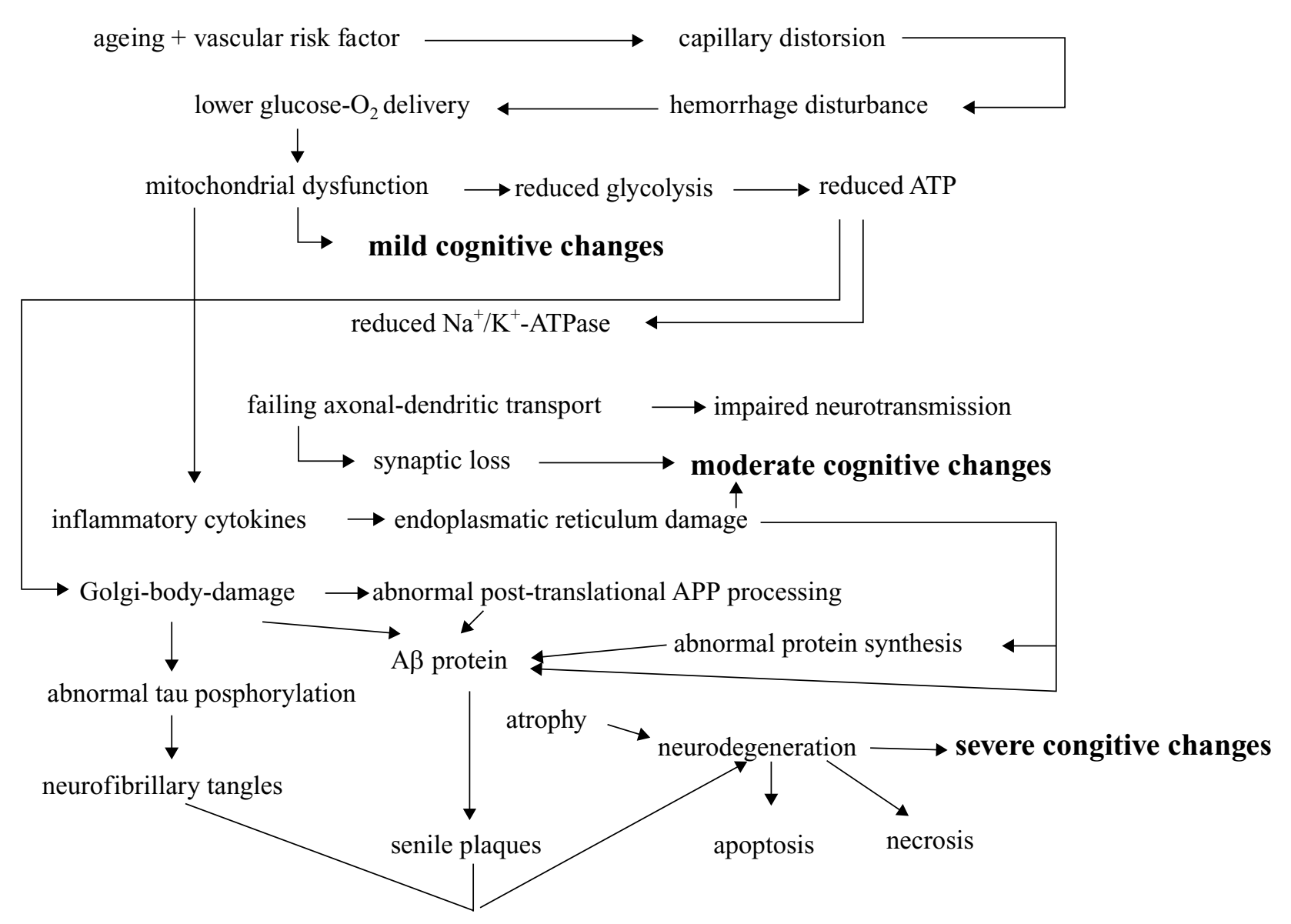

Figure $\mathbf{5}$ Evolution of sporadic Alzheimer's disease in view of aging and vascular risk factor. Abbreviation: APP, amyloid precursor protein.

Cholinergic deficits in $\mathrm{VaD}$ are due to ischemia of basal forebrain nuclei and of cholinergic pathways and can be treated with the use of the AchE inhibitors used in AD (Erkinjunttti et al 2004). Since the response in AD to AchE inhibitors might differ according to the magnitude of the cerebrovascular contribution, estimating the vascular burden might also help to understand the variability of the response of $\mathrm{AD}$ patients to drug treatment (Erkinjunttti et al 2004). For isolated subcortical CVD, a particular clinical picture does not characterize its association with $\mathrm{AD}$, and the additional diagnostic potential of IT is huge again (Jellinger 2005). Knowledge is lacking in determining the contribution of subcortical CVD to cognitive impairment alone or in addition to AD (Honig et al 2005). Notably, the impact of number, size and type of lesions on cognitive impairment is unclear (Honig et al 2005). A recent finding supporting this hypothesis is that corpus callosum atrophy -a proxy of global axonal loss - is strongly associated with cognitive impairment independently of any effect of white matter lesions depicted on T2 weighted MRI (Pantel et al 1998). Moreover, lacunes and other white matter lesions may have different impacts on cognitive impairment, as the first consists pathophysiologically of complete interruption of axonal fibers on pathology, whereas the second is related to incomplete infarction and demyelination (Lazarus et al 2005). Lastly, not all white matter changes detected by imaging techniques are alike (Lazarus et al 2005). Patchy areas of hypodensity in the white matter on CT and irregular and confluent white matter hyperintensities on MRI have been suggested to be due to ischemia, whereas other types of hyperintensities seem to be related more often to nonischemic changes (Lazarus et al 2005). Punctuate lesions can be an early stage of confluent lesions, but sometimes there seems to be no pathological substrate (Lazarus et al 2005). Diffuse and homogenous periventricular hypodensity of the white matter on $\mathrm{CT}$ and smooth periventricular hyperintensities on MRI are due to an increased water content, demyelination, and subependymal gliosis caused by nonischemic alterations 
of the periventricular fluid dynamics (Lazarus et al 2005). It was found that diffusion anisotropy which correlates with the apparent diffusion coefficient (ADC) mapping in MRI, did reflect mental ability and was reflected by physiological aging when a series of measurements was performed in the centrum semiovale $-\mathrm{a}$ brain region that at least in part contains watershed areas (Lövblad et al 2004). However, the altered fractional anisotrpy and ADC values in the genu of the corpus callosum of $\mathrm{AD}$ patients presumably reflect the microscopic changes of white matter degeneration in $\mathrm{AD}$ and vary with disease severity (Kantarci et al 2002; Lövblad et al 2004).

While diffusion-weighted MR imaging (DWI) is used primarily for the investigation of stroke by using the strong sensitivity to ischemia (Kantarci et al 2002; Lövblad et al 2004), the ADC maps that can be generated from the same image sets seem to contain further information regarding tissular pathophysiology: indeed this has been demonstrated in both animal and now in human models of stroke where the ADC seems not just to reflect the presence or absence of a lesion but to correlate with the underlying hemodynamic pathophysiology (Lövblad et al 2004). The changes associated with $\mathrm{AD}$ are particularly interesting because diffusion tensor imaging (DTI) within the white matter may be abnormal in the early stages of $\mathrm{AD}$ and has the potential to monitor the course of the disease (Muller et al 2005). Such MRI can give information very similar to that obtained by SPECT and PET to detect cortical atrophy and are very sensitive early indicators of hippocampal dysfunction (Yamamoto et al 1980; Muller et al 2005). The combination of macro- and microstructural imaging parameters areas could therefore be promising in early detection of neurodegenerative processes (Muller et al 2005). The main potential advantages of DWI over SPECT or PET include that this IT does not use radioactive isotopes, has superior spatial resolution and can be done in a single session with structural MRI, thereby reducing total examination time in detecting the water diffusivity caused by neuropathological change in $\mathrm{AD}$ and vascular dementia.

\section{Differentiating Alzheimer's disease from other forms of degenerative dementia}

Some infrequent types of dementia have a relatively specific imaging pattern and, in conjunction with typical clinical findings, can be easily distinguished from $\mathrm{AD}$ approaching the accuracy of neuropathology (Chong et al 2005). The morphological pattern perhaps most specific is that of extremely severe frontopolar and temporopolar atrophy of the cortical layer and white matter association of the frontal (ballooning) and temporal horns (Chetelat et al 2005). Recent advances in imaging $\mathrm{AP}$, one of the hallmarks of $\mathrm{AD}$, offer a technological breakthrough and the possibility for more efficient assessment of antiamyloid interventions as well as specific noninvasive diagnostic capabilities (Mathis et al 2005).

DWI-MRI is sensitive to microscopic motion of water molecules in cerebral tissue (Lövblad et al 2004). Its primary application has been in the diagnostic evaluation of acute cerebral ischemia (Lövblad et al 2004). The IT has also been applied to study patients with $\mathrm{AD}$, postulating that pathological destruction of cell membranes, with subsequent loss of myelin and axonal processes due to Wallerian degeneration, increases the water diffusion in the hippocampus and afferent white matter tracts (Steffens et al 2003). Such pathological changes manifest by an increased apparent DWI; DTI enables measurement of directionality or asymmetry of microscopic water movement in tissue (Kantarci et al 2002, 2005; Lövblad et al 2004). Such asymmetric diffusion, known as anisotropy, is seen in normal white matter, since the integrity of white matter tracts that allows diffusion of water preferentially parallel, rather than perpendicular, to the tracts (Steffens et al 2003). Recently, the IT has been applied in human studies of AD (Steffens et al 2003; Kantarci et al 2005) indicating that physiological aging without dementia is characterized by significant changes in the white matter most prominently in anterior brain regions (Steffens et al 2003; Head et al 2004). The dissociation between the regional effects of aging and dementia suggests that the mechanisms underlying age-associated cognitive decline are distinct from those underlying AD dementia (Head et al 2004).

The mild increase in OEF seen in AD can be interpreted as evidence of participation of the vascular factors in the reduction of energy metabolism (Nagata et al 2002). Since vascular reactivity and vascular transit time were well preserved in $\mathrm{AD}$, the mild increase of OEF is associated with vascular involvement possibly at the level of capillary and BBB, but not at the level of arterioles (Nagata et al 2002).

\section{Magnetic resonance spectroscopy}

Magnetic resonance spectroscopy (MRS) is an application of MRI that allows noninvasive assessment of a number of local metabolite in brain tissue (Lin et al 2005). The presence of altered membrane metabolism in $\mathrm{AD}$ creates opportunities for both phosphorus and proton MRS investigations of brain chemistry where conventional structural imaging appears normal (Kantarci et al 2002). Phosphorous MRS allows the simultaneous observation of a number of membrane phospholipid precursors and breakdown products; a loss of 
membrane integrity would likely change the relative ratio of these compounds compared to their levels in healthy tissue (Valenzuela et al 2001; Leybaert 2005). Less robust evidence using phosphorous MRS has shown significant phosphocreatine decline and increments in the cell membrane phosphomonoesters in the early, and possibly asymptomatic, stages of the disease (Valenzuela et al 2001). Significant relationships between measured metabolites and cognitive indices in patients with mild cognitive impairment and AD have been reported (Chantal et al 2002). In addition, phosphodiester concentration has been found to correlate strongly with AD plaque counts (Leybaert 2005). MRS of AD has therefore introduced new pathophysiologic speculations (Valenzuela et al 2001). Studies of automated MRS for AD diagnosis have reported high sensitivity and moderate specificity, but are yet to test prospective samples (Valenzuela et al 2001). As described earlier in this review, selective decreases in temporoparietal perfusion have also been associated with the diagnosis of AD using PET (Jagist 2001). Since cerebral metabolism is closely coupled to $\mathrm{CBF}$, these brain regions might demonstrate altered bioenergetic indices, which could be detected using phosphorus MRS (Valenzuela et al 2001). In contrast to consistent findings of the in vitro studies with respect to membrane biochemistry, the findings in vivo are extremely variable for AD (Jones et al 2004): these studies suggest a possible role for MRS in early diagnosis and for surrogate biochemical markers for monitoring disease progression and therapeutic response.

Proton MRS allows in vivo assessment of different metabolites like $N$-acetylaspartate (NAA), glutamine and glutamate, GABA, myo-inositol (Ins), glycine, mobile choline moieties, creatine $(\mathrm{Cr})$ and phosphocreatine, lipids, and lactate (Heminghaus et al 2003). $\mathrm{N}$-acetylaspartate is present primarily in neurons, but not in glial cells or other nonneuronal tissue (Leybaert 2005). Although the exact metabolism of $N$-acetylaspartate remains unclear, $N$-acetylaspartate is generally thought to represent a marker of neuronal function (Leybaert 2005). The $N$-acetlyaspartate level is decreased in cases of neuronal loss or damage yet but may return to normal levels during recovery suggesting a possible re-synthesis of the $\mathrm{N}$-acetylaspartate pool (Signoretti et al 2002). Elevated myo-inositol levels mark gliosis, membrane dysfunction, and/or cytoskeletal abnormalities (Heminghaus et al 2003). Elevated choline levels reflect cellular proliferation or myelin breakdown (Heminghaus et al 2003). In both vascular dementia and $\mathrm{AD}$, ratios of NAA/Cr were decreased, which may be attributed to neuronal degeneration and loss. The Ins/ $\mathrm{Cr}$-ratios were increased indicating either enhanced gliosis or alteration of the cerebral inositol metabolism (Huang et al 2001; Signoretti et al 2002; Leybaert 2005). However, the topographical distribution of the metabolic alterations in both diseases differed, revealing a temporoparietal pattern for AD and a global, subcortically pronounced pattern for vascular dementia (Huang et al 2001; Heminghaus et al 2003). Furthermore, patients suffering from vascular dementia had remarkably enhanced TMA (tetramethylammonium)/Cr ratios, potentially due to ongoing degradation of myelin (Heminghaus et al 2003). These metabolite concentrations do not necessarily correlate with disease severity (Huang et al 2001).

With regard to patients at risk of $\mathrm{AD}, N$-acetylaspartate and myo-inositol metabolite levels in patients with mild cognitive impairment are between those of cognitively normal subjects and patients with AD (Heminghaus et al 2003). Patients who are positive for the apolipoprotein $\varepsilon 4$ allele tend to demonstrate similar findings prior to the onset of dementia or notable anatomical changes (Wahlund et al 2000; Mathis et al 2005). Longitudinal evaluation may allow determining prognosis and treatment in such at-risk patients. Also, MRS measurements have been shown to be a predictor of cognitive scores at follow-up in $\mathrm{AD}$, but not in vascular dementia (Wahlund et al 2000). Furthermore, few controlled studies have used MRS to monitor treatment effects of muscarinic agonists and AchE inhibitors, demonstrating $N$-acetylaspartate measured by MRS combined with hippocampal volumetry, to provide a highly useful surrogate marker of $\mathrm{AD}$ progression in trials of neuroprotective agents (Hossain et al 2002; Krishnan et al 2003).

The model proposed for the coupling between neuronal activity and $\mathrm{CMR}_{\mathrm{glc}}$ on the basis of studies at the cellular level would be consistent with an initial glycolytic processing of glucose occurring in astrocytes during NA, resulting in a transient lactate overproduction that is shuttled to axons where it is metabolized oxidatively to generate ATP, followed by a recoupling phase during which lactate would be oxidized by neurons (Mostert et al 2004). The spatio-temporal "window" during which a lactate peak could be detected by MRS would depend on the velocity and degree of recoupling between astrocytic glycolysis and neuronal oxidative phosphorylation (Mostert et al 2004). The possible role of lactate in dementia is currently under investigation: there seems to be experimental evidence in animal models that a parallel lactate and pyruvate increase reflects a severe but not a light cognitive impairment (Pugliese et al 2005).

Difficulties remain in the clinical application - since MRS data acquisition from the whole brain is technically not 
feasible, and findings reported in studies may differ between particular regions (Kasischke et al 2004). It is also important for future MRS studies to clarify the spatial distribution of assessed metabolites and interaction between metabolites (Leybaert 2005).

\section{Neuronuclear imaging in dementia assessment}

Hundreds of patients with clinically diagnosed and in some cases histopathologically confirmed AD have been studied using PET measurements of $\mathrm{CBF}$ and $\mathrm{CMR}_{\mathrm{glc}}$ or $\mathrm{CMR}_{\mathrm{O} 2}$ (Jagust 2005) finding (i) a consistent pattern of focally decreased NA identified with PET that involves neocortical association areas, but spares basal ganglia, thalamus, cerebellum, and the cortex processing primary sensory and motor functions (Mielke et al 1996; Herholz et al 1999); (ii) the extent of hypometabolism has been correlated with the severity of cognitive impairment and often shows right/left hemispheric asymmetry (Mielke et al 1996; Herholz et al 1999); (iii) a decreased global CBF but normal oxygen metabolism (Wolf et al 1997); (iv) an increased striatal but not cortical metabolism (Rougement et al 1984). The "classic" regional cerebral pattern of bi-parietotemporal hypometabolism has been found to be associated with a high likelihood of $\mathrm{AD}$, and typical patterns have also been identified for many of the other entities, which may clinically resemble AD, but are distinguished histopathologically (Mielke et al 1996). Blinded clinical evaluations of PET are capable of differentiating patients with $\mathrm{AD}$ from patients with other dementia or from cognitively intact persons (Mielke et al 1991). Effects of normal aging on brain function have also been examined with PET. Recent measurements using FDG-PET identified an age-related decline of glucose metabolism in healthy adults, most consistently in frontal cortex; studies of carefully selected subjects find declines to be minimal in glucose metabolism throughout most of the brain areas during physiological aging (Zuchner et al 2004). (18)F-FDG PET indicates that AD-related processes lead to an altered functional relationship between the entorhinal cortex and several cortical and limbic regions, with respect to normal aging (Mosconi et al 2004). These results suggest that the assessment of coupled $\mathrm{CMR}_{\mathrm{glc}}$ reductions between the entorhinal cortex and the ipsilateral inferior temporooccipital cortex, besides the typical pattern of cortical reduction, could increase $\left[{ }^{18} \mathrm{~F}\right]$-FDG PET diagnostic sensitivity and further underline its inclusion in the clinical assessment of $\mathrm{AD}$ in the near future (Mosconi et al 2004). However, the relatively low spatial resolution of PET may hinder the detection of pathological elements in small anatomic structures, such as a layer of the entorhinal cortex or subdivision of the hippocampus.

Single photon emission computed tomography (SPECT) has historically been the most widely available FIT, and has become the one most commonly employed in the clinical evaluation of dementia (Mirzaei et al 2004). Most SPECT are perfusion-based. Although the specific radiopharmaceuticals and technique differ from those used in PET, the principles of interpretation, as well as the neurobiological processes underlying its use, are similar. The practical differences in its application are (i) a typically worse spatial resolution of SPECT compared with PET, and (ii) an occasional uncoupling between cortical metabolism (usually measured with PET) and perfusion in the presence of certain CVD. Thus, AD studies utilizing SPECT yielded results similar to those using PET, but with less sensitivity and overall accuracy (Ota et al 2005). That expectation has been further underlined through side-by-side comparisons of the two modalities, including studies performed in AD with mild symptoms, and with highresolution SPECT and PET (Silverman et al 2004).

A recent study assessing the accuracy of SPECT in early dementia followed patients longitudinally for a minimum of 2 years after obtaining SPECT with ${ }^{99 m}$ TC-HMPAO (Kemp et al 2003). Comparing the SPECT of one group progressing to probable $\mathrm{AD}$, and the other remaining stable for at least two years, both subgroups matched for mildness at time of presentation, the progression group can be distinguished from the stable group with a sensitivity of $78 \%$ and a specificity of $71 \%$ (Kemp et al 2003). A similarly designed study with PET has recently found that progression and nonprogression patients could be distinguished on an average of 3.0 years in advance with a sensitivity of $93 \%$ and a specificity of $74 \%$ (Nestor et al 2003). The higher diagnostic and prognostic sensitivity of PET may be especially relevant in identifying the disease in its earliest stages, in order to target patients for therapy when neurodegeneration is minimal (Nestor et al 2003). PET may have high diagnostic accuracy, but adding it to the standard diagnostic regimen of AD would yield limited, if any, benefits at very high costs (McMahon et al 2003).

Genetic risk factors for $\mathrm{AD}$ have been identified. The $\varepsilon 4$ allele of the apolipoprotein $\mathrm{E}$ gene is associated with a significantly increased risk of developing AD of senile onset; overall, those with the $\varepsilon 4 / \varepsilon 3$ or $\varepsilon 4 / \varepsilon 4$ genotype are more than twice as likely to have AD compared with individuals with the $\varepsilon 3 / \varepsilon 3$ genotype (Mosconi et al 2005). FDG-PET have linked the $\varepsilon 4$ allele to hypometabolism in the posterior cingulated, parietal, and temporal cortex, and have identified 
greater metabolic asymmetry in nondemented relatives of individuals with probable AD (Lee et al 2003). Furthermore, significant metabolic decline in these regions has been observed in those who have inherited the $\varepsilon 4$ allele measured by repeated PET in the same subjects over a 2-year interval (Lee et al 2003).

The majority of PET in the memory-impaired population used FDG as a radiolabeled tracer. Most recently, a new radiolabeled tracer, known as $2-\left(1-\left\{6-\left[2-\left[{ }^{18} \mathrm{~F}\right]\right.\right.\right.$ fluoroe thyl(methyl)amino]-2naphthiyl \}ethylidene)malononitrile or $\left[{ }^{18} \mathrm{~F}\right]$ FDDNP, has been developed (Nordberg 2004). This tracer is reported to target the amyloid- $\beta$ senile plaques and neurofibrillary tangles in AD (Nordberg 2004). Additional ligands are in the preclinical development phase.

\section{Neuronuclear imaging of neurotransmitter system The acetylcholine system}

A direct demonstration of cholinergic receptor function, however, may provide a method to select patients for treatment (Herholz et al 2004; Zuchner et al 2005) as cholinergic mechanisms have been shown to control amyloid precursor protein processing and the number of muscarinic M2-acetylcholine receptors is decreased in brain regions of patients with $\mathrm{AD}$ enriched with senile plaques (Zuchner et al 2005). Similarly, demonstration of treatment effects on neurochemical changes of cellular levels by IT will be important for the refinement of pharmacotherapy in dementia (Smith et al 2003). Both, presynaptic deficits to determine the possible need for cholinergic therapy and post-synaptic deficits to assess the likelihood of successful response have to be studied (Herholz et al 2004). Two SPECT ligands are currently used: (i) iodobezovesamicol (IBVM) as a marker of ACh terminal density, which has successfully demonstrated marked reduction in patients with NINCDS diagnosed probable AD (Kuhl et al 1996). Studies have demonstrated that brain regions showing IBVM deficits in $\mathrm{AD}$ are similar to those showing reductions in $\mathrm{CMR}_{\mathrm{glc}}$ and CBF using PET or SPECT (Kuhl et al 1996). ${ }^{123} \mathrm{I}-(\mathrm{R}, \mathrm{S}) \mathrm{QNB}$ (iodine-labeled 3-quinuclidinyl benzilate) or ${ }^{123} \mathrm{I}-(\mathrm{R}, \mathrm{R}) \mathrm{QNB}$ binds to muscarinic receptors (Wang et al 2005) and has been used to show that there is little receptor density reduction in patients with mild AD but varying degrees of reduction in moderate-to-severe AD.

\section{The dopamine system}

Specific SPECT ligands for dopamine transporter (FP-CIT, $\beta$-CIT, IPT, TRODAT) provides a marker for presynaptic neuronal degeneration (Marshall et al 2003). Striatal uptake correlates with disease severity, in particular bradykinesia and rigidity, and monitoring of progression assists in clinical trials of potential neuroprotective drugs (Marshall et al 2003). The dopamine transporter ligands $\beta$-CIT and FP-CIT are of particular interest in dementia, and both are labeled with iodine-123 (Donnemiller et al 1997). The main difference between these compounds is the recommended imaging time, which is 24 hours post-injection for $\beta$-CIT and around $5 \mathrm{~h}$ for FP-CIT (Marshall et al 2003). Although the most obvious use of these compounds is the early detection of Parkinson disease and differentiation from similar presentations such as essential tremor and vascular parkinsonism, another likely application is the differentiation of DLB from AD (Marshall et al 2003): abnormal results in DLB, normal in $\mathrm{AD}$. Features of DLB, the second most common cause of degenerative dementia, include progressive cognitive deterioration, often associated with levodopa-unresponsive parkinsonism, fluctuations of cognitive functions, and psychotic symptoms (visual and auditory hallucinations, depression) (Marshall et al 2003). In a study of both CBF and dopamine transporter binding (using $\beta$-CIT), a subtle difference in perfusion patterns was found and a greater degree of occipital hypoperfusion was found in DLB as compared with AD (Donnemiller et al 1997).

\section{Techniques for imaging amyloid- $\beta$ in vivo}

Imaging of $\mathrm{AD}$ progression would greatly facilitate the discovery of therapeutics, and a wide range of ligands are currently under investigation for the AP detection by PET (Lockhard et al 2005) (see Table 1 and Table 2).

Table I Comparison of techniques for in vivo imaging of amyloid $\beta$ (Bacskai et al 2002)

\begin{tabular}{llll}
\hline Technique & $\begin{array}{l}\text { Cross-sectional } \\
\text { resolution }(\mathbf{m m})^{1)}\end{array}$ & $\begin{array}{l}\text { Ionizing } \\
\text { radiation }\end{array}$ & Volume \\
\hline PET & $2-6$ & Yes & Entire brain \\
SPECT & $1-10$ & Yes & Entire brain \\
MRI & $0.1-1$ & No & Entire brain \\
Multiphoton & 0.001 & No & No \\
\hline
\end{tabular}

Abbreviations: PET, positron emission tomography; SPECT, single photon emission computed tomography; MRI, magnetic resonance imaging; mm, millimetre. 
Table 2 Agents for in vivo imaging of amyloid- $\beta$ (Bacskai et al 2002)

\begin{tabular}{lll}
\hline Imaging agent & $\begin{array}{l}\text { Imaging } \\
\text { technique }\end{array}$ & Signal-to-noise ratio \\
\hline Thioflavin & Multiphoton & High \\
Thiazine Red $\mathrm{R}$ & Multiphoton & High \\
MeO-XO & Multiphoton & High \\
BTA-I & Multiphoton & High \\
{$\left[{ }^{18} \mathrm{~F}\right] \mathrm{FDDNP}$} & PET & Low \\
$\mathrm{A} \beta$ peptides & Multiphoton & Moderate \\
Antibodies & Multiphoton & Very high \\
\hline
\end{tabular}

Abbreviations: BTA-I, thioflavin T; PET, positron emission tomography; FDDNP, [(2-fluoroethyl)(methyl) amino]-2-naphthyl]ethzlidene)malnonitrile.

The requirements for successful amyloid detection and quantification include the availability of an AP-specific radioligand that incorporates a PET radionuclide within its structure (Lockhart et al 2005). As spatial resolution of PET is relatively low, PET is unable to detect individual receptors, PET will not depict individual plaques (Lockhart et al 2005). Instead, PET should provide a quantification of the amyloid density in different brain regions and demonstrate differences between regions containing high and low densities of AP (Lockhart et al 2005). AP-Imaging can provide objective measures of preclinical disease state, when its changes in the follow-up are measured serially (Ono et al 2005). Such information can be used in prevention trials or in monitoring of progression and effectiveness of AD treatment.

Different strategies are used to develop compounds suitable for AP-IT in humans (Ono et al 2005). Monoclonal antibodies against AP or peptide fragments have limited the up-take by the brain when tested in patients with AD if tested for immune therapy of AD (Geylis et al 2005). When putrescine-gadolinium- $\beta$-amyloid has been injected into transgenic mice over-expressing amyloid, labeling has been observed with MRI (Vanhoutte et al 2005) creating an opportunity for the noninvasive investigation of the longitudinal development of the plaques in animals and perhaps humans. The small molecular approach for AP-IT has so far been most successful (Higuchi et al 2005). The binding of different derivatives of Congo red and thioflavin has been studied in human autopsy brain tissue and in transgenic mice (Nordberg 2004). Agents that recognize AP should improve imaging contrast and detection sensitivity (Higuchi et al 2005). Suitable agents for detection of AP must meet several criteria: (i) the compound must bind to AP with high specificity; (ii) the reagent must pass the BBB in sufficient amounts to allow detection (Maccioni et al 2004). This requires that the compound is relatively small and lipophilic
(Nordberg 2004). The reagent should bind with high affinity to allow retention of the compound specifically bound to AP while the free and nonspecifically bound compound clears from brain (Maccioni et al 2004). Two compounds, fluorine-18-labelled-FDDNP and carbon-11-labelled-PIB, both show more binding in the brains of patients with $A D$ than in those of healthy people (Nordberg, 2004). Attempts have been made to detect amyloid using $\left[{ }^{18} \mathrm{~F}\right]$ FDDNP, labeled senile plaques and perineuronal nets in tissue sections from AD brain; however, specificity for these neuropathological lesions is not clear (Agdeppa et al 2001). Perineuronal nets are a specialized form of extracellular matrix and consist of large aggregating chondroitin sulfate proteoglycans, with hyaluronan and tenascin as main components, which surround subpopulations of neurons (Morcarski et al 2004). The glycosaminoglycan components of perineuronal nets form highly charged structures in the direct microenvironment of neurons and thus might be involved in local ion homeostasis (Morcarski et al 2004).

Taken together, perineuronal nets store associative memory, do not involve long-term potentiation, and are also loci for AD pathophysiology contributing to or causing early memory loss in clinically manifested AD (Alkon 1999). PET revealed good BBB permeability, and a sufficient bioavailability of different radiolabelled probes in $\mathrm{AD}$ patients as compared to controls (Agdeppa et al 2001; Niwa et al 2002). Unfortunately, nonspecific accumulation of the very lipophilic $\left[{ }^{18} \mathrm{~F}\right] \mathrm{FDDNP}$ is high in white matter areas (Nordberg 2004). $\left[{ }^{18} \mathrm{~F}\right]$ FDDNP initially accumulates most in the pons (an area without significant numbers of plaques or tangles) and least in the hippocampus (an area of severe pathology) (Nordberg 2004).

Besides the first steps of ${ }^{19} \mathrm{~F}$ and ${ }^{1} \mathrm{H}$ MRI using a fluorine-containing probe in animals (Higuchi et al 2005), no method that does not involve artificially breaking the BBB has captured AP in vivo with sufficient sensitivity and specificity throughout the brain in a definitive manner that could be confirmed by subsequent immunohistochemestry. Continuing engineering efforts to improve spatial resolution are underway in MRI, which may enable IT of individual plaques with contrast probes (Paduslo et al 2002) enabling early diagnosis and also provide a direct measurement of the efficacy of antiamyloid therapies currently being developed.

\section{Microscopy}

In vitro studies indicate that amyloid plaques contain multiple binding sites that can accommodate structurally diverse 
compounds, providing flexibility for radiopharmaceutical design of AP imaging agents (Mathis et al 2004). Compared with large biomolecules, small molecule radiotracers are often readily accessible through chemical synthesis and can display superior brain permeability (Christie et al 2001). Several small molecule AP-imaging radioligands display high binding affinities to AP and sufficient brain penetration for imaging studies (Bacskai et al 2003; D'Amore et al 2003). Very specific detection of AP can be obtained with antibodies targeted against AP (D'Amore et al 2003). Antibodies enter the CNS in very small amounts when administered peripherally but have not been successfully used for IT yet (Christie et al 2001). However, fluorescently labeled anti-AP antibodies were applied directly to the brains of transgenic mice and were detected with multiphoton microscopy (D'Amore et al 2003). The antibodies labeled AP within a few hundred micrometers from the surface of the cortex with high sensitivity and specificity (Bacskai et al 2003). Surprisingly, also a rapid clearance of the labeled AP has been demonstrated, confirming the usability of an immunotherapeutic approach to treat AD (Christie et al 2001). It is possible that manipulations that increase BBB permeability for labeled antibodies would further improve IT.

Multiphoton microscopy allows very high resolution $(<0.001 \mathrm{~mm})$ imaging of microsopic structures with high sensitivity (Bacskai et al 2003). This light microscope-based technique depends on detecting fluorescence (either autofluorescence or from a contrast reagent), though no radioactive tracers are necessary (Christie et al 2001) using a pulsed nearinfrared laser to excite fluorescence for deeper penetration than visible light microscopy not scattering tissue, and optical tomography with restricted excitation volume (Christie et al 2001). The technique depends on an invasive technique to allow access to the brain, and imaging depths of about $500 \mu \mathrm{m}$ are achievable leading to a very small imaging volume within the cortex compared to the size of the brain (Christie et al 2001). Fluorescent contrast reagents with high specificity result in very high contrast images of immunohistochemically defined microscopic structures (Christie et al 2001; Bacskai et al 2003) allowing direct examination of the natural history of plaques and evaluation of antiplaque therapeutics in models of AD (Bacskai et al 2002, 2003).

In vivo imaging has been performed with fluorescent derivatives of thioflavin T and Congo red (Nordberg 2004). Both compounds have been used as histochemical stains for fibrially amyloid- $\beta$ deposits: They are small, lipophilic derivates and enter the brain rapidly. Multiphoton microscopy is used to identify the labeled plaques in transgenic mouse brain
$30 \mathrm{~min}$ after intravenous injection or $24 \mathrm{~h}$ after intraperitoneal injection (D'Amore et al 2004). The fluorescent reagents specifically labeled senile plaques and cerebrovascular amyloid (Nordberg 2004). They have near ideal properties for AP-imaging, and multiphoton microscopy demonstrates that these compounds label immunohistochemically defined AP in transgenic mice over-expression of mutant human amyloid precursor protein (D'Amore et al 2004). Radiolabel led versions of methody-XO4 and BTA-1 may also be excellent candidates for PET in AD (Schmidt et al 2001; Kleindienst et al 2004).

\section{Functional imaging methods other than single photon emission tomography and positron emission tomography}

FMRI offers a method to indirectly measure functional brain changes associated with NA (Goekoop et al 2004, 2006) being similar in its use to PET (Nair 2005). Both methods are able to indirectly measure NA in individual patients because of changes in blood properties (Nair 2005). The main type of fMRI is the blood oxygen level dependent (BOLD) imaging which does not involve the injection of paramagnetic contrast agents and is based on differences in the paramagnetic properties between oxy- and deoxyhemoglobulin to detect regional differences in blood oxygenation, usually in comparison between a NA and a resting task (Rombouts et al 2005). BOLD contrast MRI is a simple noninvasive method of estimating "perfusion", and combined with a vasodilatory stimulus, may allow estimation of cerebral vascular reserve (Nag 2003). BOLD signal change appears to originate from net changes in averaged NA areas of cortex that trigger, in some manner, $\mathrm{CVC}$ leading to the measured response (Nag 2003). A question directly relevant to imaging AD is whether this coupling is intact and if it is possible to make quantitative comparisons between individuals and groups (Gonzalez 1996).

In addition, pharmacological fMRI may help to examine neurochemical changes - ie, of the cholinergic system (Goekoop et al 2004) - in early disease of AD by studying signal reactivity to pharmacological challenge at cellular levels. In addition, a recent study using fMRI showed increased brain activation during memory tasks in APOE-4 carriers in brain regions affected by AD (Small 2002). Such pharmacological MRI have implications for understanding the neurochemical mechanism by which cognitive enhancing medications exert these effects on brain function and for the use of fMRI in early detection and treatment monitoring of AD (Goekoop et al 2006). 
Table 3 Use of different imaging methods to investigate the neurovascular mechanism in Alzheimer's disease (Lee et al 2003)

\begin{tabular}{|c|c|}
\hline Technique & Indications and role \\
\hline \multicolumn{2}{|c|}{ Routine examinations } \\
\hline CT/MRI & Exclusion of disease, anatomy \\
\hline MR volumetrics & Hippocampal atrophy \\
\hline \multicolumn{2}{|c|}{ Semiroutine examinations } \\
\hline SPECT/PET & Global/regional blood flow, metabolic changes \\
\hline DWI & Global/regional dysfunction \\
\hline \multicolumn{2}{|l|}{ Research } \\
\hline MR volumetrics & $\begin{array}{l}\text { Frontal/temporal lobe atrophy, whole brain atrophy; } \\
\text { sequential: rate of volume loss }\end{array}$ \\
\hline MRS & Neuronal loss, abnormal metabolite \\
\hline DTI & Neuronal integrity \\
\hline fMRI & Memory, associative functional dysfunction \\
\hline
\end{tabular}

Abbreviations: $C T$, computed tomography; $M R I$, magnetic resonance imaging; $M R$, magnetic resonance; SPECT, single-photon-emission computed tomography; PET, positron emission tomography; DWI, diffusion weighted imaging; MRS, magnetic resonance spectroscopy; DTI, diffusion tensor imaging; fMIR, functional magnetic resonance imaging.

FMRI and examining brain-behavior relationships are recent IT that contribute to dementing illness and are notably improving our understanding of the pathophysiology of the different subgroups of dementia (Goekoop et al 2006). The results of fMRI of cognitively impaired or at-risk patients have shown increases or decreases in the intensity and extent of NA, as compared with findings in control groups (Johnson et al 2005). The discrepancies between increased or decreased NA may have to do with either differing task demands or different levels of compensatory reserve (Goekoop et al 2006). In addition, the differential response to cholinergic challenge in mild cognitive impairment and $\mathrm{AD}$ visualized by fMRI may reflect a difference in the functional status of the cholinergic system between both subgroups, which is in line with recent results showing a differential clinical response to cholinergic treatment (Goekoop et al 2006).

Three kinds of fMRI to study AD have emerged, all still in their developmental stages. One approach focuses on examining tasks, such as encoding information into recent memory, that are known to be compromised in AD (Goekoop et al 2006). A second approach examines activation patterns that result from the performance of tasks that are completed successfully but may be more difficult in patients at risk for AD. The idea behind such an approach is that cognitive tasks can serve as "stress tests", with individuals at the earliest stages of the disease requiring more neural activity to reach the same level of performance (Hotz 2001). A final approach, which has been used in only a few preliminary studies performed to this point, is to image tasks that yield downstream markers of medial temporal function (Buckner et al 2000).

\section{Diagnostic evaluation}

CVD and stroke relate to a high risk of cognitive impairment, but also relate to AD: vascular cognitive impairment and dementias extend beyond the traditional multi-infarct dementia (Table 3). The pathophysiology of vascular dementias includes interactions between vascular etiologies (CVD and vascular risk-factors), changes in the brain (infarcts, white matter lesions, atrophy), host factors (age) and cognition (Schaller 2004a). Variation in defining the cognitive syndrome, in vascular etiologies, and allowable brain changes in current criteria have resulted in variable estimates of prevalence, of subgroups of subjects, and of the subtypes and distribution of putative causal brain lesions (Lee et al 2003).

Subcortical ischemic vascular disease and dementia incorporate small vessel disease as the main vascular etiology, lacunar infarct and ischemic white matter lesions as primary type of brain lesions, subcortical location as the primary location of lesions, and subcortical syndrome as the primary clinical manifestation (Solerte et al 2002). It incorporates two clinical entities, "Binswanger's disease" and "the lacunar state" (Jellinger 2002). AD with vascular dementias (mixed dementia) has been underestimated as a prevalent cause in the older population (Jellinger 2005). In addition to simple co-existence, vascular dementia and $\mathrm{AD}$ have closer interaction: several vascular risk factors and vascular brain changes relate to clinical manifestation of $\mathrm{AD}$, and they also share common pathogenetic mechanisms (Langa et al 2004). Vascular cognitive impairment is a category aiming to replace the "Alzheimerized" dementia concept in the setting of CVD, and substitute it with a spectrum that includes subtle cognitive deficits of vascular origin, post-stroke dementia, and the complex group of the vascular dementias (Erkinjunttti et al 2004; Mirzaei et al 2004). So far, there is no standard treatment for vascular dementias, and still little is known about the primary prevention (brain at risk for CVD) and secondary prevention (CVD-brain at risk for vascular cognitive impairment/vascular dementia) (Roman et al 2000). Future focus should be directed to the distinct etiological and pathological factors: the vascular and the $\mathrm{AD}$ burden of the brain; the state-of-the-art-imaging, and especially the molecular imaging, has a main place in this research. 


\section{Early detection of Alzheimer's disease}

PET has been used to directly quantify several processes relevant to the status of cerebral health and function, including $\mathrm{CBF}$, cerebral blood volume, $\mathrm{CMR}_{\mathrm{O} 2}$, and $\mathrm{CMR}_{\mathrm{glc}}$ demonstrating diagnostic and prognostic use in evaluating patients who have cognitive impairment, and in distinguishing among primary neurodegenerative dementias and other causes of cognitive decline (Silverman et al 2005). These PET found, that patients with AD may differentially express resting $\mathrm{CBF}$ covariance patterns even at very early disease stages (Scarmeas et al 2004). Expression of covariance patterns correlates with cognitive and functional performance measures, holding promise for meaningful associations with underlying biopathological processes (Eustache et al 1997; Herholz et al 2000).

Ishii and colleagues $(2000,2001)$ demonstrated deficits in perfusion and metabolism, using ${ }^{18} \mathrm{FDG}$ and $\mathrm{H}_{2}{ }^{15} \mathrm{O}$ PET in mild $\mathrm{AD}$ : at the mild and moderate stages of $\mathrm{AD}$, glucose metabolism is reduced not only in the parietotemporal region but also in the posterior cingulate and precuneus. At the advanced stage of $\mathrm{AD}$, there is also a metabolic reduction in the frontal region (Herholz et al 2000). In AD patients, $\mathrm{CMR}_{\text {glc }}$ is relatively preserved in the pons, sensorimotor cortices, primary visual cortices, basal ganglia, thalamus and cerebellum (Herholz et al 2000). Recent investigations revealed that asymptomatic members of $\mathrm{AD}$ families have significant parietal and temporal deficits compared to control subjects, and that nondemented people in their mid-fifties with increased genetic AD risk, as conferred by apolipoprotein E allele $\varepsilon($ apoE-4), have significantly lower parietal metabolism than those without the apoE-4 allele (Mosconi et al 2005). Other brain regions typically affected by AD have also been reported to be hypometabolic in individuals homo- or heterozygous for apoE-4, including posterior cingulate, inferior parietal, temporal, and prefrontal regions (Mosconi et al 2005).

\section{Differential diagnosis of Alzheimer's disease}

Neuropathology and structural MRI have pointed to the medial temporal lobe as the brain region earliest affected in AD (Jellinger 2002; Hund-Georgiadis et al 2003). T1weighted MRI findings provide strong evidence that in mild cognitive impairments, AD-related volume losses can be reproducibly detected in the hippocampus, the entorhinal cortex and, to a lesser extent, the parahippocampal gyrus; they also indicate that lateral temporal lobe changes are becoming increasingly useful in predicting the transition to dementia (Mosconi 2005). The 3-D data acquisition in MRI enables exact calculation of volumes and coregistration of images during follow-up examination (Barkhof 2003). Perfusion MRI may be performed to demonstrate areas of reduced $\mathrm{CBV}$ and $\mathrm{CBF}$ in vascular dementia and several forms of degenerative brain disorders (Barkhof 2003). The CBF reduction is multifocal and subcortical in vascular dementia and shows a cortical distribution in AD (Barkhof 2003). DWI and DTI of individuals with AD demonstrate changes in whole brain white matter and posterior fiber tract with some evidence suggesting that the posterior fiber tracts are more affected than anterior fiber tract (Kantarci et al 2001). The pathophysiological basis of DTI effects remains uncertain. In addition, current experimental data suggests that magnitude and BOLD brain response in activated particular brain regions is different in AD (Bondi et al 2004). In addition, a diamine- and gadolinium-substituted derivative of $\beta$-amyloid is shown to have enhanced in vitro binding to $\mathrm{AD} A \mathrm{AP}$ and increased in vivo permeability at $\mathrm{BBB}$ because of the unique Asp/Glu substitutions (Paduslo et al 2004). Because of MRI contrast enhancement provided by gadolinium, MRI of individual $\mathrm{AP}$ in $\mathrm{AD}$ brains allows for early diagnosis to other dementia subgroups and also provides a direct measure of the efficacy of anti-AP therapies currently being developed (Vanhoutte et al 2005).

Failure of BBB with leakage of serum components into and through the walls of cerebral small vessels leading to neuronal and glial damage may be an important common mechanism in $\mathrm{AD}$ and vascular dementia (Zlokovic et al 2004). Together with an intravenous paramagnetic contrast agent, such as gadolinium-DTPA, MRI can also detect and quantify BBB damage (Vlassenko et al 2006). Further studies are required to prove the hypothesis that $\mathrm{BBB}$ failure and perforating artery endothelial leakage might be a common pathogenetic mechanism for lacunar stroke, white matter hyperintensities, and dementia. If so, new treatments could be targeted toward this new pathophysiological mechanism to prevent the development of or to reduce BBB leakage.

FDG-PET has revealed glucose metabolic reductions in the parieto-temporal, frontal and posterior cingulate cortices to be the hallmark of AD (Mosconi 2005). Overall, the pattern of cortical metabolic changes has been useful for the prediction of future $\mathrm{AD}$ as well as in distinguishing AD from other neurodegenerative diseases (Mosconi 2005). FDG-PET on average achieves $90 \%$ sensitivity in identifying $\mathrm{AD}$, although specificity in differentiating $\mathrm{AD}$ from other dementias is lower (Mosconi 2005). Moreover, recent MRIguided FDG-PET have shown that medial temporal lobe 
hypometabolism is the most specific and sensitive measure for the identification of mild cognitive impairments, while the utility of cortical deficits is controversial (Mosconi 2005).

\section{Comparative accuracy of positron emission tomography}

Since PET have shown that psychological or cognitive activation in mild-to-moderate $\mathrm{AD}$ can increase $\mathrm{CBF}$ and $\mathrm{CMR}_{\mathrm{glc}}$ as in normal subjects, a similar finding with BOLD fMRI would demonstrate not the quantity but the topographic pattern of activation to differentiate $\mathrm{AD}$ from normal persons.

Although the accuracy of the diagnosis is of critical importance, only few studies addressed this question by providing data of sensitivity and specificity following a representative patient mix (Kodama et al 2002). The sensitivity of other diagnostic methods is substantially lower than reported by PET, although the specificity is comparable (Herholz et al 2000; Mielke et al 2001).

In vivo proton MRS enables investigation of specific metabolites within tissues. A comparison between ${ }^{31}$ phosphorus MRS and FDG-PET in AD was unable to find a correlation between phosphorus metabolic and either glucose metabolic or dementia severity; hence, measurement of $\mathrm{rCMR}_{\mathrm{glc}}$ represents a promising technique, but with uncertain clinical specificity and utility (Mielke et al 2001) suggesting that in AD reduced neuronal energy performance and membrane abnormalities contribute to metabolic deterioration (Mielke et al 2001).

\section{Prospects for the future}

PET and SPECT has come to be quite useful for assessing therapeutical effects of newly introduced AD treatment (Matsuda 2001). A number of questions still need to be addressed; in particular the different time constants for synaptic activity and the metabolic response as the basis of the signals detected with PET need to be considered (Matsuda 2001). In this context, it is likely that the glutamate-mediated coupling at neuron-astrocyte metabolic units in an activated cortical area will be compounded through a mechanism in which the extracellular space acts as a spatial and temporal metabolic buffer (Matsuda 2001). The usefulness of these methods in the clinical routine is promising but there are no data that demonstrate whether they contribute a diagnostic value yet.

Different molecular IT may be useful not only to diagnose different subtypes of dementia but also to monitor therapeutic efforts (Raszkiewicz et al 1992). For example, carriers of a common $\mathrm{AD}$ susceptibility gene have functional brain abnormalities in young adulthood, several decades before the possible onset of dementia. With the increasing options to treat dementia symptoms in the future, this aspect may gain increased interest, and IT may become more and more the target of experimental research (Raszkiewicz et al 1992).

\section{Conclusion}

Cerebral arteriosclerosis or stroke may increase the deposition of AP or the formation of neurofibrillary tangles. Alternatively, they may simply hasten the age at onset of disease, or increase the severity of disease symptoms. Disruption of this regulatory network occurs in response to brain injury, for example, during stroke. Evidence now indicates that cerebrovascular dysfunction is at least an aggravating, concomitant feature of neurodegenerative disorders such as AD. Accumulation of $\mathrm{AP}$ is associated with neuronal death in $\mathrm{AD}$. AP induces dysfunction in all cell types of the neurovascular unit and interferes with the function of blood vessels. These effects are mediated through reactive oxygen species. This raises the provocative question of whether $\mathrm{AD}$ is secondary to vascular dysfunction in the CNS and, therefore, the neurodegeneration that follows is a consequence of inadequate $\mathrm{CBF}$, altered brain metabolism and failure in physiological functions of brain endothelium, which represents a site at the BBB.

$\mathrm{AD}$ IT opens the window to a new direction for experimental research and clinical diagnosis or treatment of $\mathrm{AD}$. This approach could be the basis of longitudinal studies in experimental models of AD to search for biomarkers associated with AP pathologies as well as to track disease course after treatment with candidate pharmacological substances.

\section{References}

Agdeppa ED, Kepe V, Liu J, et al. 2001. Binding characteristics of radiofluorinated 6-dialkylamino-2-naphthylethylidene derivates as positron emission tomography imaging probes for beta-amyloid plaques in Alzheimer's disease. J Neurosci, 21:RC189.

Alexander E, Wagner E, Buchner D, et al. 1995. Do surgical brain lesions present as isolated dementia? A population based study. J Am Geriatr Soc, 43:138-43.

Alkon DL. 1999. Ionic conductance determinants of synaptic memory nets and their implications for Alzheimer's disease. $J$ Neurosci Res, 58:24-32.

American Academy of Neurology. 1994. Practice parameter for diagnosis and evaluation of dementia. Report of the Quality Standards Subcommittee of the American Academy of Neurology. Neurology, 44:2203-6.

Ames III A. 2000. CNS energy metabolism as related to function. Brain Res Brain Res Rev, 34:42-68.

Ances BM, Greenberg JH, Detre JA. 2000a. Effects of variations in interstimulus interval on activation-flow coupling response and somatosensory evoked potentials with forepaw stimulation in the rat, $J$ Cereb Blood Flow Metab, 20:290-7.

Ances BM, Zarahn E, Greenberg JH, et al. 2000b. Coupling of neuronal activation to blood flow in the somatosensory cortex of rats is time-intensity separable, but not linear. J Cereb Blood Flow Metab, 20:921-30. 
Anderson B, Rutledge V. 1996. Age and hemispheric effects on dendritic structure. Brain, 119:1983-90.

Atochin DN, Demchenko IT, Astern J, et al. 2003. Contribution of endothelial and neuronal nitric oxide synthase to cerebrovascular responses to hypoxia. J Cereb Blood Flow Metab, 23:1219-26.

Ayata C, Ma J, Meng W, et al. 1996. L-NA-sensitive rCBF augmentation during vibrissal stimulation in type III nitric oxide synthase mutant mice. J Cereb Blood Flow Metab, 16:539-41.

Back T. 1998. Pathophysiology of the ischemic penumbra - revision of a concept. Cell Mol Neurobiol, 18:621-38.

Bacskai BJ, Hickey GA, Skoch J, et al. 2003. Four-dimensional multiphoton imaging of brain entry, amyloid binding, and clearance of an amyloid-beta ligand in transgenic mice. Proc Natl Acad Sci USA, 100:12462-7.

Bacskai BJ, Hyman BT. 2002. Alzheimer's disease: what multiphoton microscopy teaches us. Neuroscientist, 8:386-90.

Bakker SL, deLecuar FE, denHeijer T, et al. 2004. Cerebral haemodynamic in the elderly. The Rotterdam study. Neuroepidemiology, 23:178-84.

Barbelivien A, Noel C, MacKenzie ET, et al. 1999. Cerebrovascular evidence for a GABAergic modulation of the cholinergic vasodilatory basalocortical system in the rat. Brain Res, 834:223-7.

Barkhof F. 2003. Guidelines for brain imaging in vascular dementia clinical trials. Int Psychogeriatr, 15(Suppl 1):273-6.

Baron JC, Godeau C. 2000. Human aging. In: Toga A, Mazziotta JC, eds. Brain mapping: the systems. San Diego, CA: Acad Pr., pp. 591-604.

Bartus RT, Dean RL, Beer B, et al. 1982. The cholinergic hypothesis of geriatric memory dysfunction. Science, 217:408-14.

Baumach GL, Sigmund CD, Faraci FM. 2004. Structure of cerebral arterioles in mice deficient in expression of the gene for endothelial nitric oxide synthase. Circ Res, 95:822-9.

Bernardinelli Y, Magistretti PJ, Chatton JY. 2004. Astrocytes generate Na+ mediated metabolic waves, Proc Natl Acad Sci USA, 101:14937-42.

Blanc EM, Toborek M, Mark RJ, et al. 1997. Amyloid- $\beta$-peptide induces cel monolayer albumin permeability, impairs glucose transport, and induces apoptosis in vascular endothelial cells. J Neurochem, 68:1870-81.

Blin J, Piercey MF, Giuffra ME, et al. 1994. Metabolic effects of scopolamine and physiostigmine in human brain measured by positron emission tomography. J Neurol Sci, 123:4-51.

Bondi MW, Houston WS, Eyler LT, et al. 2005. fMRI evidence of compensatory mechanism in older at genetic risk for Alzheimer disease Neurology, 64:501-8.

Bonvento G, Sibson N, Pellerin L. Does glutamate image your thought? Trends Neurosci, 25:359-64.

Browne SE, Ayata C, Huang PL, et al. 1999. The cerebral metabolic consequences of nitric oxide synthase deficiency: glucose utilization in endothelial and neuronal nitric oxide synthase null mice. $J$ Cereb Blood Flow Metab, 19:144-8.

Buckner RL, Snyder AZ, Sanders AL, et al. 2000. Functional brain imaging of young, nondemented, and demented older adults. J Cogn Neurosci, 12(Suppl 2):24-34.

Buxton RB, Uludag K, Dubowitz DJ, et al. 2004. Modelling the hemodynamic response to brain activation. Neuroimage, 23(Suppl 1)S220-33.

Camanduola S, Poli G, Mattson MP. 2000. The lipid peroxidation product 4-hydorxy-2,3,-nonenal, inhibits constitution and inducible activity of nuclear factor kappa B in neurons. Brain Res Mol Brain Res, 85:53-60.

Cauli B, Tong XK, Rancillac A, et al. 2004. Cortical GABA interneurons in neurovascular coupling: relays for subcortical vasoactive pathways. J Neurosci, 24:8940-9.

Chalmers K, Wilcock GK, Love S. 2003. APOE epsilon 4 influences the pathological phenotype of Alzheimer's disease by favouring cerebrovascular over parenchyma accumulation of A beta protein. Neuropathol Appl Neurobiol, 29:231-8.

Chantal S, Labelle M, Bouchard RW, et al. 2002. Correlation of regional proton magnetic resonance spectroscopy metabolic changes with cognitive deficits in mild Alzheimer disease. Arch Neurol, 59:955-62.
Chatton JV, Pellerin L, Magistretti PJ. 2003. GABA uptake into astrocytes is not associated with significant metabolic cost: Implications for brain imaging of inhibitory transmission. PNAS, 100:12456-61.

Chen XQ, Qin LY, Zhang CG, et al. 2005. Presence of neuroglobin in cultured astrocytes. Glia, 50:182-6.

Chetelat G, Landeau B, Eustache F, et al. 2005. Using voxel-based morphometry to map the structural changes associated with rapid conversion in MCI: a longitudinal MRI study. Neuroimage, 27:934-46.

Cholet N, Pellerin L, Magistretti PJ, et al. 2002. Similar perisynaptic glial localization for the $\mathrm{Na}+, \mathrm{K}+-\mathrm{ATPase}$ alpha 2 subunit and the glutamate transporters GLAST and GLT-1 in the rat somatsensory cortex. Cereb Cortex, 12:515-25.

Cholet N, Seylaz J, Lacombe P, et al. 1997. Local uncoupling of the cerebrovascular and metabolic responses to somatosensory stimulation after neuronal nitric oxide synthase inhibition. $J$ Cereb Blood Flow Metab, 17:1191-201.

Chong MS, Sahadevan S. 2005. Preclinical Alzheimer's disease: Diagnosis and prediction of progression; Lancet Neurol, 4:576-9.

Christie R, Kimchi E, Kajdasz S, et al. Multiphoton microscopy and amyloid angiopathy. Amyloid, 8(Suppl 1):48-50.

Collerton D. 1986. Cholinergic function and intellectual decline in Alzheimer's disease. Neuroscience, 19:1-28.

Csernansky JG, Wang L, Miller JP, et al. 2005. Preclinical detection of Alzheimer's disease: hippocampal shape and volume predict dementia onset in the elderly. Neuroimage, 25:783-92.

Czosnyka M, Balestreri M, Steiner L, et al. 2005. Age, intracranial pressure, autoregulation, and outcome after brain trauma, J Neurosurg, 102:450-4.

Cruz NF, Dienel GA. 2002. High glycogen levels in brain of rats with minimal environmental stimuli: implications for metabolic contributions of working astrocytes. J Cereb Blood Flow Metab, 22:1476-89.

Cummings JL. 1994. Vascular subcortical dementias: clinical aspects. Dementia, 5:177-80.

D'Amore JDS, Kajdusz ST, Mchellan ME, et al. 2003. In vivo multiphoton imaging of a transgenic mouse model of Alzheimer disease reveals marked thioflavin-S-associated alterations in neurite trajectories. $J$ Neuropathol Exp Neurol, 62:137-45.

Dauphin F, MacKenzie ET. 1995. Cholinergic and vasoactive intestinal polypeptidergic innervation of the cerebral arteries. Pharmacol Ther, 67:385-417.

Decarli C. 2004. Vascular factors in dementia: an overview. J Neurol Sci, 226:19-23.

De la Torre JC. 2002. Alzheimer disease as a vascular disorder: nosological evidence. Stroke, 33:1152-62.

De la Torre JC, Fortin T, Park GA, et al. 1992. Chronic cerebrovascular insufficiency induces dementia like deficits in aged rats. Brain Res, 582:186-95.

Desmond DW. 1996. Vascular dementia: a construct in evolution. Cerebrovasc Brain Metabol Rev, 8:296-325.

Detre JA, Wang J. 2002. Technical aspects and utility of fMRI using BOLD and ASL. Clin Neurophysiol, 113:621-34.

Dickerson BC, Sperling RA. 2005. Neuroimaging biomarkers for clinical trials of disease-modifying therapies in Alzheimer's disease. NeuroRx, 2:348-60.

Donnemiller E, Heilmann J, Wenning GK, et al. 1997. Brain perfusion szintigraphy with ${ }^{99 \mathrm{~m}} \mathrm{Tc}-\mathrm{HmPAO}$ or ${ }^{99 \mathrm{~m}} \mathrm{Tc}-\mathrm{ECD}$ and ${ }^{123}$ I-beta-CIT singlephoton emission tomography in dementia of the Alzheimer-type and diffuse Lewy body disease. Eur J Nucl Med, 24:320-25.

Duara R, Barker WW, Chang J, et al. 1992. Viability of neocortical function shown in behavioral activation state PET studies in Alzheimer disease. J Cereb Blood Flow Metab, 12:917-34.

Earnest MO, Heaton RK, Wilkinson WE, et al. 1979. Cortical atrophy, ventricular enlargement and intellectual impairment in the aged. Neurology, 29:1138-43.

Erkinjunttti T, Roman G, Gauthier S. 2004. Treatment of vascular dementia-evidence from clinical trials with cholinesterase inhibitors. Neurol Res, 26:603-5. 
Erkinjuntti T, Ostby T, Steenhuis R, et al. 1997. The effect of different diagnostic criteria on the prevalence of dementia. $N$ Engl J Med, 337:1667-74.

Eustache F, Desgranges B, Petit-Taboue MC, et al. 1997. Transient global amnesia: implicit/explicit memory dissociation and PET assessment of brain perfusion and oxygen metabolism in the acute stage. J Neurol Neurosurgy Psychiatry, 63:357-67.

Forette F, Seux ML, Staessen JA, et al.; On behalf of the Syst-Eur Investigators. 1998. Prevention of dementia in randomized double-blind placebo-controlled systolic hypertension in Europe (Syst-Eur) trial. Lancet, 352:1347-51.

Fox PT, Raichle ME. 1986. Focal physiological uncoupling of cerebral blood flow and oxidative metabolism during somatosensory stimulation of human subjects. Proc Nat Acad Sci USA, 83:1140-4.

Frachowiak RSJ, Pozilli C, Legg MJ, et al. 1981. Regional cerebral oxygen supply and utilization in dementia. Brain, 104753-78.

Fukuyama H, Ouchi Y, Matsuzaki S, et al. 1996. Focal cortical blood flow activation is regulated by intrinsic cortical cholinergic neurons, Neuroimage, 3:195-201.

Furchgott RF, Zawadzki JV. 1980. The obligatory role of endothelial cells in the relaxation of arterial smooth muscle by acetylcholine. Nature, 280:373-6.

German DC, Yazdani U, Speziale SG, et al. 2003. Cholinergic neuropathology in a mouse model of Alzheimer's disease. J Comp Neurol, 462:371-81.

Geylis V, Kourilov V, Meiner Z, et al. 2005. Human monoclonal antibodies against amyloid-beta from healthy adults, Neurobiol Aging, 26:597-606.

Giovacchini G, Lerner A, Toczek MT, et al. 2004. Brain incorporation of 11C-arachidonic acid, blood volume, and blood flow in healthy aging: a study with partial-volume correction. $J$ Nucl Med, 45:1471-9.

Goekoop R, Scheltens P, Barkhof F, et al. 2006. Cholinergic challenge in Alzheimer patients and mild cognitive impairment differentially affects hippocampal activation - a pharmacological fMRI study. Brain, 129:141-57.

Goekoop R, Rombouts SA, Jonker C, et al. 2004. Challenging the cholinergic system in mild cognitive impairment: a pharmacological fMRI study, Neuroimaging, 23:1450-9.

Gonzalez RG. 1996. Molecular and functional resonance neuroimaging for the study of dementia. Ann NY Acad, 777:37-48.

Gotoh J, Kuang TY, Nakao Y, et al. 2001. Regional differences in mechanisms of cerebral circulatory response to neuronal activation. Am J Physiol Heart Circ Physiol, 280:H821-9.

Gsell W, DeSadeleer C, Toutain J, et al. 2005. Simultaneous analysis of the coupling between cerebral blood flow, cerebral glucose use and neuronal activity in the rat during a somatosensory activation. Soc Neurosci Abs, pp. 322-3.

Gsell W, DeSadeleer C, Marchalant Y, et al. 2000. The use of cerebral blood flow as an index of neuronal activity in functional neuroimaging: experimental and pathophysiological considerations. J Chem Neuroanat, 20:215-24.

Hajdu MA, Heistad DD, Siems JE, et al. 1990. Effects of aging on mechanics and composition of cerebral arterioles in rats. Circ Res, 661747-54.

Head D, Buckner RL, Shimony JS, et al. 2004. Differential vulnerability of anterior white matter in nondemented aging with minimal acceleration in dementia of the Alzheimer type: evidence from diffusion tensor imaging. Cereb Cortex, 14:410-23.

Heminghaus S, Frolich L, Gerriz C, et al. 2003. Brain metabolism in Alzheimer disease and vascular dementia assessed by in vivo proton magnetic resonance spectroscopy, Psychiatry Res, 123:183-90.

Herholz K, Adams R, Kessler R, et al. 1990. Criteria for the diagnosis of Alzheimer's disease with positron emission tomography. Dementia, 1:156-64.

Herholz K, Bauer B, Wienhard K, et al. 2000. In-vivo measurement of regional acetylcholine esterase activity in degenerative dementia: comparison with blood flow and glucose metabolism. J Neural Transm, 107:1457-8.
Herholz K, Weisenbach S, Zundorf G, et al. 2004. In vivo study of acetylcholine esterase in basal forebrain, amygdala, and cortex in mild to moderate Alzheimer disease. Neuroimage, 21:136-43.

Higuchi M, Iwata N, Matsuba Y, et al. 2005. ${ }^{19} \mathrm{~F}$ and ${ }^{1} \mathrm{H}$ MRI detection of amyloid beta plaques in vivo. Nat Neurosci, 8:527-33.

Hillman BJ. 1997. Medical imaging in the 21st century. Lancet, 350:731-3.

Ho GJ, Drego R, Hakimian E, et al. 2005. Mechanisms of cell signaling and inflammation in Alzheimer's disease. Curr Drug Targets Inflamm Allergy, 4:247-56.

Hock C, Muller-Spahn F, Schuh-Hofer S, et al. 1995. Age dependency of changes in cerebral hemoglobin oxygenation during brain activation: a near-infrared spectroscopy study. J Cereb Blood Flow Metab, 15:1103-6.

Hof PR, Morrison JH. 2004. The aging brain: morphomolecular senescence of cortical circuits. Trends Neurosci, 27:607-13.

Honer WG, Prohovnik I, Smith G, et al. 1988. Scopolamine reduces frontal cortex perfusion. J Cereb Blood Flow Metab, 8:635-41.

Honig LS, Kukull W, Mayeux R. 2005. Artherosclerosis and Alzheimer disease: Analysis of data from the US National Alzheimer's Coordinating Centre. Neurology, 64:494-500.

Hossain M, Jhee SS, Shioritz T, et al. 2002. Estimation of the absolute bioavailability of rivastigmine in patient with mild to moderate dementia of the Alzheimer's type, Clin Pharmacokinet, 41:225-34.

Hotz RL. 2001. "Cognitive stress test" may yield early clues of preclinical AD. Am J Alzheimers Dis Other Demen, 16:9-10.

Howard EF, Chen Q, Cheng C, et al. 1998. NF-kappa B is activated and ICAM-1 gene expression is upregulated during reoxygenation of human brain endothelial cells, Neurosci Lett, 248:199-203.

$\mathrm{Hu}$ X, Le TH, Ugurbil K. 1997. Evaluation of the early response in fMRI in individual subjects using short stimulus duration. Magn Reson Med, 37:877-84.

Huang W, Alexander GE, Chang L, et al. 2001. Brain metabolite concentration and dementia severity in Alzheimer's disease: a (1)H MRS study. Neurology, 57:626-32.

Hund-Georgiadis M, Zysset S, Naganawa S, et al. 2003. Determination of cerebrovascular reactivity by means of fMRI signal changes in cerebral microangiopathy: a correlation with morphological abnormalities. Cerebrovasc Dis, 16:158-65.

Hyder F, Kennan RP, Kida I, et al. 2000. Dependence of oxygen delivery on blood flow in rat brain. J Cereb Blood Flow Metab, 20:485-98.

Iadecola C. 2004. Neurovascular regulation in the normal brain and in Alzheimer's disease. Nat Rev Neurosci, 5:347-60.

Iadecola C. 2003. Cerebrovascular effects of amyloid-beta peptides: mechanisms and implications for Alzheimer's dementia. Cell Mol Neurobiol, 23:681-9.

Ide K, Schmalbruch IK, Quistorff B, et al. 2000. Lactate, glucose and O2 uptake in human brain during recovery from maximal exercise. J Physiol, 522:159-64.

Ide K, Horn A, Secher NH. 1999. Cerebral metabolic response to submaximal exercise. J Appl Physiol, 87:1604-8.

Illes J, Kann D, Karetsky K, et al. 2004. Advertising, patients' decision making, and self referral for computed tomography and magnetic resonance imaging, Arch Intern Med, 164:2415-19.

Inoue K, Goto R, Nakagawa M, et al. 2005. Apparent CBF decrease with normal aging due to partial volume effects: MR-based partial volume correction on CBF SPECT. Ann Nucl Med, 19:283-90.

Ishii K, Willoch F, Minoshima S, et al. 2001. Statistical brain mapping of 18F-FDG PET in Alzheimer's disease: validation of anatomic standardization for atrophical brains. $J$ Nucl Med, 42:548-57.

Ishii K, Sasaki M, Matsui M, et al. 2000. A diagnostic method for suspected Alzheimer's disease using $\mathrm{H}(2) 15 \mathrm{O}$ positron emission tomography perfusion Z score. Neuroradiology, 42:787-94.

Ishimaru H, Takahashi A, Ikarashi Y, et al. 1998. NGF delays rather than prevents the cholinergic terminal damage and delayed neuronal death in the hippocampus after ischemia. Brain Res, 789:194-200.

Jagist W. 2004. Molecular neuroimaging in Alzheimer's disease. Neurorx, 1:206-12. 
Jagust W. 2005. Dementia: finding the signals in the noise. Lancet Neurol, $4: 10-11$.

Jellinger KA. 2005. Understanding the pathology of vascular cognitive impairment. J Neurol Sci, 229-30:57-63.

Jellinger KA. 2002. Alzheimer disease and cerebrovascular pathology: an update. J Neural Transm, 109:813-36.

Jellinger KA, Mitter-Festl E. 2003. The impact of cerebrovascular lesions in Alzheimer disease - a comparative autopsy study. J Neurol, 250:1050-5.

Jobst KA, Smith AD, Barker CS, et al. 1992. Association of atrophy of the medical temporal lobe with reduced blood flow in the posterior parietotemporal cortex in patients with a clinical and pathological diagnosis of Alzheimer's disease. J Neurol Neurosurg Psychiat, 55:190-4.

Johnson SC, Schmitz TW, Moritz CH, et al. 2006. Activation of brain regions vulnerable to Alzheimer's disease: The effect of mild cognitive impairment. Neurobiol Aging, 27:1604-12.

Johnson NA, Jahng GH, Weiner MW, et al. 2005. Pattern of cerebral hypoperfusion in Alzheimer diseases and mild cognitive impairmen measured with arterial spin-labelling MR imaging: initial expression. Radiology, 234:851-9.

Jones RS, Waldman AD. 2004. 1H-MRS evaluation of metabolism in Alzheimer's disease and vascular dementia. Neurol Res, 26:488-95.

Kacem K, Lacome P, Seylaz J, et al. 1998. Structural organization of the perivascular astrocyte endfeet and their relationship with the endothelial glucose transporter: a confocal microscopy study. Glia, 23:1-10.

Kalaria RN. 2003. Vascular factors in Alzheimer's disease. Int Psychogeriatr, 15(Suppl 1):47-52.

Kalaria RN, Harik SI. 1989. Abnormalities of the glucose transporter at the blood - brain barrier and in the brain in Alzheimer's disease. Prog Clin Biol Res, 317:415-21.

Kasischke KA, Vishwasrao HD, Fisher PJ, et al. 2004. Neuronal activity triggers neuronal oxidative metabolism followed by astrocytic glycolysis. Science, 305:99-103.

Kantarci K, Petersen RC, Boeve BF, et al. 2005. DWI predicts future progression to Alzheimer disease in amnestic mild cognitive impairment. Neurology, 64:902-4.

Kantarci K, Xu Y, Shiung MM, et al. 2002. Comparative diagnostic utility of different MR modalities in mild cognitive impairment and Alzheimer' disease. Dement Geriatr Cogn Disord, 14:198-207.

Kantarci K, Jack CR, Xu YC, et al. 2001. Mild cognitive impairment and Alzheimer disease: regional diffusively of water. Radiology, 219:101-7

Kemp PH, Holmes C, Hoffmann SM, et al. 2003. Alzheimer's disease: differences in technetium-99 m HMPAO SPECT scan findings between early onset and late onset dementia, J Neurol Neurosurg Psychiatry, 74:715-19.

Kleindienst A, Harvey HB, Riece AC, et al. 2004a. Intraventricular infusion of the neurotrophine protein S100 B improves cognitive recovery after fluid percussion injury in the rat. $J$ Neurotrauma, 21:541-7.

Kleindienst A, Hildebrandt G, Kroemer SA, et al. 2004b. Hypothalamic neuropeptide release after experimental subarachnoid hemorrhage: in vivo microdialysis study. Acta Neurol Scand, 109:361-8.

Klunk WE, Wang Y, Huang GF, et al. 2003. The binding of 2-(4'-methylami nophenyl)benzothiazole to postmortem brain homogenates is dominated by the amyloid component. $J$ Neurosci, 23:2086-92.

Kodama N, Shimada T, Fukumoto I. 2002. Image-based diagnosis of Alzheimer-type dementia: measurements of hippocampal and ventricular areas in MR images. Magn Reson Med Sci, 1:14-20.

Koos T, Tepper JM. 1999. Inhibitory control of neostriatal projection neurons by GABAergic interneurons. Nat Neurosci, 2:467-72.

Koistinaho M, Koistinaho J. 2005. Interactions between Alzheimer's disease and cerebral ischemia-focus on inflammation. Brain Res Brain Res Rev, 48:240-50.

Krishnan KR, Charles HC, Dorasiwamy PM, et al. 2003. Randomized, placebo-controlled trial of the effect of donepezil on neuronal markers and hippocampal volumes in Alzheimer's disease. Am J Psychiatry, 160:2003-11.
Kuhl DE, Minoshima S, Fessler JA, et al. 1996. In vivo mapping of cholinergic terminals in normal aging, Alzheimer's disease, and Parkinson's disease. Ann Neurol, 40:399-410.

Lahiri DK, Chen D, Ge YW, et al. 2003. Does nitric oxide synthase contribute to the pathogenesis of Alzheimer's disease?: effects of beta-amyloid deposition on NOS in transgenic mouse brain with AD pathology. Ann NY Acad Sci, 1010:639-42.

Langa KM, Foster NL, Larson FB. 2004. Mixed dementia: emerging concepts and therapeutic implications. JAMA, 292:2901-8.

Lauritzen M, Gold L. 2003. Brain function and neurophysiological correlates of signals used in functional neuroimaging. J Neurosci, 23:3972-80.

Lazarus R, Prettyman R, Cherryman G. 2005. White matter lesions on magnetic resonance imaging and their relationship with vascular risk factor in memory clinic attenders. Int J Geriatr Psychiatry, 20:274-9.

Lee KU, Lee JS. Kim KW, et al. 2003. Influence of the apolipoprotein E type 4 allele on cerebral glucose metabolism in Alzheimer's disease patients. J Neuropsychiatry Clin Neurosci, 15:78-83.

Leybaert L. 2005. Neurobarrier coupling in the brain: a partner of neurovascular and neurometabolic coupling? J Cereb Blood Flow Metab, 25:2-16.

Lin A, Ross BD, Harris K, et al. 2005. Efficacy of proton magnetic resonance spectroscopy in neurological diagnosis and neurotherapeutic decision making. NeuroRx, 2:197-214.

Lindauer U, Megow D, Schultze J, et al. 1996. Nitric oxide synthase inhibition does not affect somatosensory evoked potentials in the rat. Neurosci Lett, 216:207-10.

Lindauer U, Megow D, Matsuda H, et al. 1999. Nitric oxide: a modulator, but not a mediator of neurovascular coupling in rat somatosensory cortex. Am J Physiol, 277:H799-811.

Lockhart A, Ve L, Judd DB, et al. 2005. Evidence for the presence of three distinct binding sites for the thioflavin T class of Alzheimer's disease PET imaging agents on (beta)-amyloid peptide fibrils. $J$ Biol Chem, 280:7677-84.

Lövblad KO, Delavelle J, Wetzel S, et al. 2004. ADC mapping of the aging frontal lobes in mild cognitive impairment, Neuroradiology, $46: 282-6$.

Longstreth WT, Manolio TA, Arualold A, et al. 1996. Clinical correlates of white matter findings on cranial magnetic resonance imaging of 3301 elderly people: the cardiovascular healthy study. Stroke, 27:1274-82.

Maccioni RB, Lavados M, Maccioni CB, et al. 2004. Biological markers of Alzheimer's disease and mild cognitive impairment. Curr Alzheimer Res, 1:307-14.

Mackowiak-Cordoliani MA, Bombois S, Memin A, et al. 2005. Poststroke dementia in the elderly, Drugs Agingi, 22:483-93.

Magistrettti PJ. 2000. Cellular bases of functional brain imaging: insights from neuron-glia metabolic coupling. Brain Res, 886:108-12.

Magistretti PJ, Pellerin L, Rothman DL, et al. 1999. Energy on demand. Science, 283:496-7.

Mark RE, Griffin ST, Graham DI. 1997. Aging-associated changes in human brain. J Neuropathol Exp Neurol, 56:1269-75.

Masawa N, Yoshida Y, Yamada T, et al. 1994. Morphometry of structural preservation of turcica media in aged and hypertensive human intracerebral arteries. Stroke, 25:122-7.

Marshall V, Grosset D. 2003. Role of dopamine transporter imaging in routine clinical practice, Mov Disord, 18:1415-23.

Massman PJ, Bigler ED, Cullum CM, et al. 1986. The relationship between cortical atrophy and ventricular volume. Int J Neurosci, 30:87-99.

Mathiesen C, Caesar K, Akgoren N, et al. 1998. Modification of activitydependent increases of cerebral blood flow by excitatory synaptic activity and spikes in rat cerebellar cortex. J Physiol (Lond), 512:555-66.

Mathis CA, Klunk WE, Price JC, et al. 2005. Imaging technology for neurodegenerative diseases: Progress toward detection of specific pathologies. Arch Neurol, 62:196-200.

Mathis CA, Wang J, Klunk WE. 2004. Imaging beta-amyloid plaques and neurofibrillary tangles in the aging human brain. Curr Pharm Dis, 10:1469-92. 
Matsuda H. 2001. Cerebral blood flow and metabolic abnormalities in Alzheimer's disease. Ann Nucl Med, 15:85-92.

Mayahn WG, Faraci FM, Baumbauch GL, et al. 1990. Effects of aging on responses of cerebral arterioles. Am J Physiol, 258:H1138-43.

McMahon PM, Araki SS, Sandberg EA, et al. 288. Cost-effectiveness of PET in the diagnosis of Alzheimer disease. Radiology, 228:515-22.

Mesulam MM, Mufson EJ, Levey AI, et al. 1983. Cholinergic innervation of cortex by the basal forebrain: cytochemistry and cortical connections of the septal area, diagonal band nuclei, nucleus basalis (substantia innominata), and hypothalamus in the rhesus monkey. J Comp Neurol, 214:170-97.

Meyer JS, Chowdhury MH, Xu G, et al. 2002. Donepezil treatment of vascular dementia. Ann N Y Acad Sci, 977:482-6.

Mielke R, Schopphoft HH, Kugel H, et al. 2001. Relation between 1H MR spectroscopic imaging and regional cerebral glucose metabolism in Alzheimer's disease, Int J Neurosci, 107:233-45.

Mielke R, Schroeder R, Fink GR, et al. 1996. Regional cerebral glucose metabolism and postmortem pathology in Alzheimer's disease. Acta Neuropathol (Berlin), 91:174-9.

Mielke R, Herholz K, Grond M, et al. 1991. Differences of regional cerebral blood glucose metabolism between presenile and senile dementia of the Alzheimer type, Neurobiol Aging, 13:93-8.

Minoshima S, Giordani B, Berent S, et al. 1997. Metabolic reduction in the posterior cingulated cortex in very early AD. Ann Neurol, 42:85-94.

Mirzaei S, Gelpi E, Booij J, et al. 2004. New approaches in nuclear medicine for early diagnosis of Alzheimer's disease. Curr Alzheimer Res, 3:219-29.

Moody DM, Brown WR, Chala VR, et al. 1997. Cerebral microvascular alterations in aging, leukariosis, and Alzheimer's disease. Ann NY Acad Sci, 826:103-16.

Morcarski M, Bruckner MK, Riederer P, et al. 2004. Perineuronal nets potentially protect against oxidative stress. Exp Neurol, 188:309-15.

Mosconi L. 2005. Brain glucose metabolism in the early and specific diagnosis of Alzheimer's disease FDG-PET studies in MCI and AD. Eur J Nucl Med Mol Imaging, 32:486-516.

Mosconi L, Herholz K, Prohovnik I, et al. 2005. Metabolic interaction between Apo E genotype and onset age in Alzheimer's disease: implications for brain reserve. J Neurol Neurosurg Psychiatry, 76:15-23.

Mosconi L, Pupi A, De Cristofaro MT, et al. 2004. Functional interaction of the entorhinal cortex: an 18F-FDG PET study on normal aging and Alzheimer's disease. J Nucl Med, 45:382-92.

Mostert JP, Sijens PE, Oudkerk M, et al. 2005. (1)H magnetic resonance spectroscopy of the internal capsule in human brain: a feasibility study to detect lactate following contralateral motor activity. Eur Radiol, 15:1349-52.

Mufson EJ, Ginsberg SD, Ikonomovic MD, et al. 2003. Human cholinergic basal forebrain: chemoanatomy and neurologic dysfunction. $J$ Chem Neuroanat, 26:233-42.

Mullan M, Crawford F. 1993. Genetic and molecular advances in Alzheimer's disease. Trends Neurosci, 16:398-403.

Muller MJ, Greverus D, Dellani PR, et al. 2005. Functional implications of hippocampal volume and diffusivity in mild cognitive impairment. Neuroimage, 28:1033-42.

Nag S. 2003. Morphological and molecular properties of cellular components of normal cerebral vessels. Methods Mol Med, 89:3-36.

Nagata K, Sato M, Sath Y, et al. 2002. Hemodynamic aspects of Alzheimer's disease. Ann NY Acad Sci, 977:391-402.

Nagata K, Buchan RJ, Yokoyama E, et al. 1997. Misery perfusion with preserved vascular reactivity in Alzheimer's disease. Ann NY Acad Sci, 26:272-81.

Nair DG. 2005. About being BOLD. Brain Res Brain Res Rev, 50:229-43.

Nestor PJ, Caine D, Fryer TD, et al. 2003. The topography of metabolic deficits in posterior cortical atrophy (the visual variant of Alzheimer's disease) with FDG-PET. J Neurol Neurosurg Psychiatry, 74:1521-9.

Niwa K, Kuzama K, Younkin L, et al. 2002a. Cerebrovascular autoregulation is profoundly impaired in mice overexpressing amyloid precursor protein. Am J Physiol Heart Circ Physiol, 283:H315-23.
Niwa K, Kazama K, Younkin SG, et al. 2002b. Alterations in cerebral blood flow and glucose utilization in mice overexpression the amyloid precursor protein. Neurobiol Dis, 9:61-8.

Nordberg AS. 2004. PET imaging of amyloid in Alzheimer's disease. Lancet Neurol, 3:519-27.

Ogawa M, Fukuyama H, Ouchi Y, et al. 1996. Altered energy metabolism in Alzheimer's disease. J Neurol Sci, 139:78-82.

Ono M, Yoshida N, Ishibashi K, et al. 2005. Radioiodinated flavones for in vivo imaging of beta-amyloid plaques in the brain. $\mathrm{J} \mathrm{Med} \mathrm{Chem,}$ 48:7253-60.

Ota T, Shinotoh H, Fukushik K, et al. 2005. A simple method for the detection of abnormal brain regions in Alzheimer's disease patients using (11 C) MP4A: comparison with (123I) IMP SPECT. Ann Nucl Med, 18:187-93.

Paduslo JF, Curran GL, Peterson JA, et al. 2004. Design and chemical synthesis of a magnetic resonance contrast agent with enhanced in vitro binding, high blood - brain barrier permeability, and in vivo targeting to Alzheimer's disease amyloid plaques. Biochemistry, 43:6064-75.

Paduslo JF, Wengenack TM, Curran GL, et al. 2002. Molecular targeting of Alzheimer's amyloid plaques for contrast-enhanced magnetic resonance imaging. Neurobiol Dis, 11:315-29.

Pantel J, Schroder J, Essig M, et al. 1998. Corpus callosum in Alzheimer's disease and vascular dementia - a quantitative magnetic resonance study. J Neural Transm Suppl, 54:129-36.

Paris B. 1997. The utility of CT scanning in diagnostic dementia. Mount Sinai J Med, 64:372-5.

Paul RH, Haque O, Gunstad J, et al. 2005. Subcortical hyperintensities impact cognitive function among a select subset of healthy elderly. Arch Clin Neuropsychol, 20:697-704.

Pietrini P, Alexander GE, Furey ML, et al. 2000. Cerebral metabolic response to passive audiovisual stimulation in patients with Alzheimer's disease and healthy volunteers assessed by PET. J Nucl Med, 41:575-83.

Pellerin L, Magistretti PJ. 1994. Glutamate uptake into astrocytes stimulates aerobic glycolysis: a mechanism coupling neuronal activity to glucose utilization, Proc Natl Acad Sci USA, 91:10625-9.

Peruzzi P, Lacombe P, Moro V, et al. 1993. The cerebrovascular effects of physiostigmine are not mediated through the substantia innominata. Exp Neurol, 122:319-26.

Pratt RD, Perdomo CA; Donepezil VaD 307 and 308 Study Groups. 2002. Donepezil-treated patients with probable vascular dementia demonstrate cognitive benefits. Ann NY Acad Sci, 977:513-22.

Pohjasvaara T, Mantyla R, Ylikoski R, et al. 2000. Comparison of different clinical criteria (DSM-III, ADDTC, ICD-10, NINDS-AIREN, DSM-IV) for the diagnosis of vascular dementia. National Institute of Neurological Disorders and Stroke-Association Internationale pour la Recherche et l'Enseignement en Neuroscience. Stroke, 31:2952-7.

Poirier J. 2000. Apolipoprotein E and Alzheimer's disease. A role of amyloid catabolism. Ann NY Acad Sci, 924:81-90.

Postiglione A, Lassen NA, Holeman BL. 1993. Cerebral blood flow in patients with dementia of Alzheimer's type. Aging (Milano), 5:19-26.

Poulin P, Zakzanis KK. 2002. In vivo neuroanatomy of Alzheimer's disease: evidence from structural and functional brain imaging. Brain Cogn, 49:220-5.

Pugliese M, Carrasco JL, Andrade C. 2005. Severe cognitive impairment correlates with higher cerebrospinal fluid levels of lactate and pyruvate in a canine model of senile dementia. Prog Neuropsychopharmacol Biol Psychiatry, 29:603-10.

Ranicillac A, Rossier J, Guille M, et al. 2006. Glutamatergic control of micorvascular tone by distinct GABA neurons in the cerebellum. $J$ Neurosci, 26:6997-7006.

Raszkiewicz JL, Linville DG, Kerwin Jr JF, et al. 1992. Nitric oxide synthase is critical in mediating basal forebrain regulation of cortical cerebral circulation. J Neurosci Res, 33:129-35.

Reiman EM, Chen K, Alexander GE, et al. 2004. Functional brain abnormalities in young adults at genetic risk for late onset Alzheimer's disease. Proc Natl Acad Sci USA, 101:284-9. 
Riddle Dr, Sonntag WE, Lichtenwalner RJ. 2003. Microvascular plasticity in aging. Ageing Res Rev, 2:149-168.

Riecker A, Grodd W, Klose U, et al. 2003. Relation between regional functional MRI activation and vascular reactivity to carbon dioxide during normal aging. J Cereb Blood Flow Metab, 23:565-73.

Rodrigo J, Fernandez-Vizarra O, Castro-Blanco S, et al. 2004. Nitric oxide in the cerebral cortex of amyloid-precursor protein (JW) Tg2576 transgenic mice. Neuroscience, 128:73-89.

Roland PE, Eriksson L, Stone-Elander S, et al. 1987. Does mental activity changes to oxidative metabolism of the brain? J Neurosci, 7:2373-89.

Roman GC, Sachdev P, Royall DR, et al. 2004. Vascular cognitive disorder: a new diagnostic category updating vascular cognitive impairment and vascular dementia. J Neurol Sci, 226:81-7.

Rombouts Sa, Goekoop R, Stam CJ, et al. 2005. Delayed rather than decreased BOLD response as a marker for early Alzheimer's disease. Neuroimage, 26:1078-85.

Rosengarten B, Paulsen S, Molnar S, et al. 2006. Acetylcholine esterase inhibitor donepezil improves dynamic cerebrovascular regulation in Alzheimer patients. J Neurol, 253:58-64.

Ross AJ, Sachdew PS, Wen W, et al. 2005. (1)H MRS in stroke patients with and without cognitive impairment. Neurobiol Aging, 26:873-82.

Rougement D, Baron JS, Collard P, et al. 1984. Local cerebral glucose utilization in treated and untreated patients with Parkinson's disease J Neurol Neurosurg Psychiatr, 47:824-30.

Ruitenberg A, den Heijer T, Bakker SL, et al. 2005. Cerebral hypoperfusion and clinical onset of dementia: the Rotterdam Study. Ann Neurol, 57:789-94.

Sackett DL, Haynes RB. 2002. The architectures of diagnostic research. $B M J, 324: 539-41$.

Sandor P. 1999. Nervous control of the cerebrovascular system: doubts and facts. Neurochem Int, 35:237-59.

Scarmeas N, Habeck CG, Zaruhu E, et al. 2004. Covariance PET patterns in early Alzheimer's disease and subjects with cognitive impairment but not dementia: utility in groups discrimination and correlation with functional performance. Neuroimage, 23:35-45.

Schaller BJ. 2007. Influence of age on stroke and preconditioning-induced ischemic tolerance in the brain. Exp Neurol, 205:9-19.

Schaller BJ, Modo M, Buchfelder M. 2007. Molecular imaging of brain tumors: A bridge between clinical and molecular medicine? Mol Imaging Biol, 9:60-71.

Schaller BJ, Buchfelder M. 2006. Neuroprotection in primary brain tumors: sense or nonsense? Expert Rev Neurother, 6 2006:723-30.

Schaller BJ, Bahr M, Buchfelder M. 2005. Pathophysiology of brain ischemia: penumbra, gene expression, and future therapeutic options. Eur Neurol, 54:179-80.

Schaller B. 2005a. Introduction to serial reviews on free radicals and stroke. Free Radic Biol Med, 38:409-410.

Schaller B. 2005b. Prospects for the future: the role of free radicals in the treatment of stroke. Free Radic Biol Med, 38:411-25.

Schaller B. 2004a. Usefulness of positron emission tomography in diagnosis and treatment follow-up of brain tumors. Neurobiol Dis, 15:437-48.

Schaller B. 2004b. Physiology of cerebral venous blood flow: from experimental data in animals to normal function in humans. Brain Res Brain Res Rev, 46:243-260.

Schaller B, Graf R. 2004a. Cerebral venous infarction: the pathophysiological concept. Cerebrovasc Dis, 18:179-88.

Schaller B, Graf R. 2004b. Cerebral ischemia and reperfusion: the pathophysiologic concept as a basis for clinical therapy, JCereb Blood Flow Metab, 24 2004b:351-371.

Schaller B, Graf R, Jacobs AH. 2003. Ischemic tolerance: a window to endogenous neuroprotection? Lancet, 362:1007-8.

Scheltens P, Fox N, Barkhof F, et al. 2002. Structural magnetic resonance imaging in the practical assessment of dementia: beyond exclusion Lancet Neurol, 1:13-21.

Schmidt B, Braun HA, Narlawar R. 2005. Drug development and PET-diagnostic for Alzheimer's disease. Curr Med Chem, 12:1677-95.
Seigneur J, Kroeger D, Nita DA, et al. 2006. Cholinergic action on cortical glial cells in vivo. Cereb Cortex, 16:655-68.

Silverman DH. 2004. Brain 18F-FDG PET in the diagnosis of neurodegenerative dementias: comparisons with perfusion SPECT and with clinical evaluations lacking nuclear imaging. J Nucl Med, 45:594-607.

Silverman DH, Alavi A. 2005. PET imaging in the assessment of normal and impaired cognitive function. Radiol Clin North Am, 43:67-77.

Simpson IA, Chundu KR, Davies-Hill T, et al. 1994. Decreased concentrations of GLUT1 and GLUT3 glucose transporters in the brains of patients with Alzheimer's disease. Ann Neurol, 35:546-51.

Signoretti S, Marmarou A, Fatouros P, et al. 2002. Application of chemical shift imaging for measurement of NAA in head injured patients. Acta Neurochir Suppl, 81:373-5.

Skoog I. 1998. Status of risk factors for vascular dementia. Neuroepidemiology, 17:2-9.

Slosman DO, Chicherio CH, Ludwig C. et al. 2001. ${ }^{133}$ Xe SPECT cerebral blood flow study in a healthy population: determination of T-scores. J Nucl Med, 42:864-70.

Small GW. 2002. Brain-imaging surrogate markers for detection and prevention of age-related memory loss. $J$ Mol Neurosci, 192:17-21.

Smith D, Pernet A, Hallett WA, et al. 2003. Lactate: a prefered fuel for human brain metabolism in vivo. J Cereb Blood Flow Metab, 23:658-64.

Smith GS, Koppel J, Goldberg S. 2003. Application of neuroreceptor imaging to psychiatry research. Psychopharmacol Bull, 37:26-65.

Snowdon DA, Greiner LH, Mortimer JA, et al. 1997. Brain infarction and clinical expression of Alzheimer's disease: the NunStudy. JAMA, $277: 813-17$

Snyder EM, Nong Y, Almeida CG, et al. 2005. Regulation of NMDA receptor trafficking by amyloid-beta. Nat Neurosci, 8:1051-8.

Sokoloff L. 1981. Relationships among local functional activity, energy metabolism, and blood flow in the central nervous system. Fed Proc, 40:2311-16

Solerte SB, Cerutti N, Mirani M, et al. 2002. Impairment of secretory pattern of IGF-1 from lymphomononuclear cells in aging and dementia of the Alzheimer's and vascular type, $J$ Endocrinol Invest, 25(10 Suppl):47-50.

Steffens DC, Taylor WD, Krishnan KR. 2003. Progression of subcortical ischemic disease from vascular depression to vascular dementia. Am J Psychiatry, 160:1751-6.

Sugihara S, Kinoshita T, Matsusue E, et al. 2004. Usefulness of diffusion tensor imaging of white matter in Alzheimer disease and vascular dementia. Acta Radiol, 45:658-63.

Sun Y, Jin K, Mao XO, et al. 2005. Effect of aging on neuroglobin expression in rodent brain. Neurobiol Aging, 26:275-8.

Tang K, Hynan LS, Baskin F, et al. 2006. Platelet amyloid precursor protein processing: A bio-marker for Alzheimer's disease. $J$ Neurol Sci, 15:53-8.

Terborg C, Gora F, Weiller C, et al. 2000. Reduced vasomotor reactivity in cerebral microangiopathy. A study with near-infrared spectroscopy and transcranial Doppler sonography, Stroke, 31 2000:924-929.

Thompson PM, Hayashi KM, Sowell ER, et al. 2004. Mapping cortical change in Alzheimer's disease, brain development, and schizophrenia. Neuroimage, 23(Suppl 1):2-S18.

Togo T, Katsuse O, Iseki E. 2004. Nitric oxide pathways in Alzheimer's disease and other neurodegenerative dementias. Neurol Res, 26:563-6.

Tsacopoulos M, Magistretti PJ. 1996. Metabolic coupling between glia and neurons, J Neurosci, 16:877-85.

Tsukada H, Kakiuchi T, Fukumoto D, et al. 2000. Docasaheaenoc acid improves the age-related impairment of the coupling mechanism between neuronal activation and functional cerebral blood flow response: a PET study in conscious monkeys. Brain Res, 862:180-6.

Ueki M, Linn F, Hossmann KA. 1988. Functional activation of cerebral blood flow and metabolism before and after global ischemia of rat brain. J Cereb Blood Flow Metab, 8:486-94.

Valenzuela MJ, Sachdev P. 2001. Magnetic resonance spectroscopy in Alzheimer diseases. Neurology, 56:592-8. 
Vanhoutte G, Dewachter I, Borghgraef P, et al. 2005. Noninvasive in vivo MRI detection of neuritic plaques associated with iron in APP(V7171) transgenic mice, a model for Alzheimer's disease. Magn Reson Med, 53:607-13.

van Laere KJ, Dierckx RA. 2001. Brain perfusion SPECT: age- and sexrelated effects correlation with voxel-based morphometric findings in healthy adults. Radiology, 221:810-17.

Vaucher E, Linville D, Hamel E. 1997. Cholinergic basal forebrain projections to nitric oxide synthase-containing neurons in the rat cerebral cortex. Neuroscience, 79:827-36.

Venton BJ, Michael DJ, Wightman RM. 2003. Correlation of local changes in extracellular oxygen and $\mathrm{pH}$ that accompany dopaminergic terminal activity in the rat caudate-putamen. $J$ Neurochem, 84:373-81.

Vlassenko AG, Rundle MM, Raichle ME, et al. 2006. Regulation of blood flow in activated human brain by cytosolic NADH/NAD+ ratio. Proc Natl Acad Sci USA, 103:1964-9.

Wahlund LO, Bronge L. 2000. Contrast-enhanced MRI of white matter lesions in patients with blood-brain barrier. Ann NY Acad Sci, 903:477-81.

Waldman AD, Rai GS. 2003. The relationship between cognitive impairment and in vivo metabolite ratios in patients with clinical Alzheimer's disease and vascular dementia: a proton magnetic resonance spectroscopy study. Neuroradiology, 45:507-12.

Wang H, Hitron IM, Iadecola C, et al. 2005. Synaptic and vascular association of neurons containing cyclooxygenase- 2 and nitric oxide synthase in rat somatosensory cortex. Cereb Cortex, 15:1250-60.

Weinberger DR, Jones D, Reba RE, et al. 1992. A comparison of FDG PET and IQNB SPECT in normal subject and in patients with dementia. $J$ Neuropsychiatr Clin Neurosci, 4:239-48.

Weller RO, Yow HY, Preston SD, et al. 2002. Cerebrovascular disease is a major factor in the failure of elimination of Abeta from the aging human brain: implications for therapy of Alzheimer's disease. Ann $N$ Y Acad Sci, 977:162-8.
Wolf T, Lindauer U, Villringer A, et al. 1997. Excessive oxygen or glucose supply does not alter the blood flow response to somatosensory stimulation or spreading depression in rats. Brain Res, 761:290-9.

Wolfson LI, Leenders KL, Brown LL, et al. 1985. Alterations of regional cerebral blood flow and oxygen metabolism in Parkinson's disease. Neurology, 35:1399-405.

Yamamoto M, Meyer JS, Sakai F, et al. 1980. Aging and cerebral vasodilator responses to hypercarbia: responses in normal aging and in person with risk factors for stroke. Arch Neurol, 37:489-96.

Yoshida T, Shiga K, Yoshikawa K, et al. 2004. White matter loss in the splenium of the corpus callosumin a case of posterior cortical atrophy: a diffusion tensor imaging study. Eur Neurol, 52:77-81.

Yoshikawa T, Murase K, Oku N, et al. 2003. Heterogeneity of cerebral blood flow in Alzheimer's disease and vascular dementia. Am J Neuroradiol, 24:1341-7.

Zanetti O, Bianchetti A, Trabucchi M. 1998. Consensus statement on Alzheimer's disease. JAMA, 279:655

Zheng D, LaMantia A-S, Purves D. 1991. Specialized vascularization of the primate visual cortex. J Neurosci, 11:2622-29.

Zlokovic BV. 2005. Neurovascular mechanisms of Alzheimer's neurodegeneration. Trends Neurosci, 28:202-8.

Zonta M, Angulo MC, Gobbo S, et al. 2003. Neuron-to-astrocyte signaling is central to the dynamic control of brain microcirculation. Nat Neurosci, 6:43-50.

Zuchner T, Schliebs R, Perez-Polo JR. 2005. Down-regulation of muscarinic acetylcholine receptor M2 adversely affects the expression of Alzheimer's disease-relevant genes and proteins. $J$ Neurochem, 95:20-32.

Zuendorf G, Kerrouche N, Herholz K, et al. 2003. Efficient principal component analysis for multivariante 3D voxel-based mapping of brain functional imaging data sets as applied to FDG-PET and normal aging. Hum Brain Mapp, 18:13-21. 